ESAIM: PROCEEDINGS, September 2007, Vol.21, 65-87

Gabriel Caloz \& Monique Dauge, Editors

\title{
UNE PERTURBATION HYPERBOLIQUE DES ÉQUATIONS DE NAVIER-STOKES
}

\author{
Marius Paicu ${ }^{1}$ And Genevit̀ve Raugel ${ }^{2}$
}

À Michel Crouzeix, avec respect, amitié et reconnaissance

\begin{abstract}
In this paper, we consider a hyperbolic perturbation of the Navier-Stokes equations in $\mathbb{R}^{n}$, $n=2,3$, given by $(0.2)$, which consists in adding the term $\varepsilon u_{t t}$ to the Navier-Stokes equations. In the case $n=2$, we recall the global existence and uniqueness of mild solutions of (0.2), for initial data in the Hilbert space $H^{1}\left(\mathbb{R}^{2}\right)^{2} \times L^{2}\left(\mathbb{R}^{2}\right)^{2}$ and appropriate forcing term $f$, when $\varepsilon>0$ is small enough, that has been proved in [16]. In the three-dimensional case, we prove a global existence result under a smallness condition of the initial data in $H^{1+\delta}\left(\mathbb{R}^{3}\right)^{3} \times H^{\delta}\left(\mathbb{R}^{3}\right)^{3}, \delta>0$, for an appropriate forcing term $f$, when $\varepsilon>0$ is small enough. This smallness condition is analogous to the one known for the global existence of strong solutions of the three-dimensional Navier-Stokes equations.
\end{abstract}

Résumé. Dans cet article, nous considérons l'équation (0.2), qui est une perturbation hyperbolique des équations de Navier-Stokes, par le terme $\varepsilon u_{t t}$. Dans le cas de la dimension deux d'espace, nous rappelons des résultats d'existence globale et d'unicité des solutions dans $H^{1}\left(\mathbb{R}^{2}\right)^{2} \times L^{2}\left(\mathbb{R}^{2}\right)^{2}$, quand $\varepsilon>0$ est suffisamment petit ( [16]). Dans le cas de la dimension trois d'espace, pour $\varepsilon>0$ suffisamment petit, nous démontrons l'existence globale de solutions intégrales sous une hypothèse de petitesse sur les données initiales dans $H^{1+\delta}\left(\mathbb{R}^{3}\right)^{3} \times H^{\delta}\left(\mathbb{R}^{3}\right)^{3}, \delta>0$ et des hypothèses adéquates sur le terme de force. Cette hypothèse de petitesse est totalement en accord avec l'hypothèse de petitesse classique pour les équations de Navier-Stokes en dimension trois.

\section{INTRODUCTION}

La version hyperbolique des équations de Navier-Stokes dans l'espace $\mathbb{R}^{n}$ tout entier, $n=2,3$ présentée ici a diverses justifications. Dans [3], dans le cas de la dimension deux d'espace, l'équation hyperbolique dissipative de Navier-Stokes (0.2) a été obtenue après relaxation des équations d'Euler et un changement de variables d'échelles. Dans [13] (voir aussi [19]), une telle équation modèle est justifiée par des arguments de relaxation dans les équations de Boltzman et des raisonnements impliquant des termes de retard. La version hyperbolique des équations de Navier-Stokes (0.2), obtenue par relaxation, est aussi utilisée en analyse numérique pour le calcul de solutions des équations de Navier-Stokes (voir [10] er [11] par exemple). Apparemment, de telles équations devraient aussi intervenir dans des modèles de formation de glace dans les lacs [9]. Remarquons aussi que cette perturbation hyperbolique des équations de Navier-Stokes est la même que la perturbation hyperbolique de l'équation de la chaleur introduite et justifiée par Cattaneo ( [4], [5]). Pour plus de détails concernant la justification des équations de Navier-Stokes hyperboliques, on pourra aussi consulter l'article [16].

\footnotetext{
1 Univ Paris-Sud, Laboratoire de Mathématiques d'Orsay, Orsay Cedex, F-91405; CNRS, Orsay cedex, F-91405

2 CNRS, Laboratoire de Mathématiques d'Orsay, Orsay Cedex, F-91405; Univ Paris-Sud, Orsay cedex, F-91405
}

(C) EDP Sciences, SMAI 2007 
Dans cet article, nous donnons des résultats d'existence globale (et d'unicité) des solutions des équations de Navier-Stokes perturbées dans $\mathbb{R}^{n}, n=2,3$ :

$$
\begin{aligned}
\varepsilon u_{\tau \tau}^{\varepsilon}+u_{\tau}^{\varepsilon}-\nu \Delta u^{\varepsilon} & =-\nabla p-u^{\varepsilon} \cdot \nabla u^{\varepsilon}+f^{\varepsilon} \\
\operatorname{div} u^{\varepsilon} & =0 \\
\left(u^{\varepsilon}, u_{\tau}^{\varepsilon}\right)(0, y) & =\left(u_{0}^{\varepsilon}(y), u_{1}^{\varepsilon}(y)\right)
\end{aligned}
$$

où la variable $u^{\varepsilon}$ désigne le champ des vecteurs vitesse, $p$ la pression et où $f$ est un terme de force pouvant dépendre du temps. Si on applique le projecteur de Leray $\mathbb{P}$ sur l'espace des vecteurs dans $L^{2}\left(\mathbb{R}^{n}\right)^{n}$ à divergence nulle, on obtient les équations

$$
\begin{aligned}
\varepsilon u_{\tau \tau}^{\varepsilon}+u_{\tau}^{\varepsilon}-\nu \mathbb{P} \Delta u^{\varepsilon} & =\mathbb{P}\left(-u^{\varepsilon} \cdot \nabla u^{\varepsilon}\right)+\mathbf{P} f^{\varepsilon} \\
\operatorname{div} u^{\varepsilon} & =0 \\
\left(u^{\varepsilon}, u_{\tau}^{\varepsilon}\right)(0, y) & =\left(u_{0}^{\varepsilon}(y), u_{1}^{\varepsilon}(y)\right)
\end{aligned}
$$

Dans [3], après avoir justifié les équations (0.2), Brenier, Natalini et Puel avaient démontré l'existence globale (et l'unicité) des solutions de (0.2) pour des données initiales dans $H^{2}\left(\mathbb{R}^{2}\right)^{2} \times H^{1}\left(\mathbb{R}^{2}\right)^{2}$, dans le cas de la dimension deux d'espace et d'une force nulle, à condition de choisir $\varepsilon>0$ inférieur à un nombre réel positif $\varepsilon_{0}$ ne dépendant que de la taille des données initiales. Ensuite, ils avaient comparé, dans la norme $L^{2}\left(\mathbb{R}^{2}\right)$, la solution de $(0.2)$ à la solution correspondante des équations de Navier-Stokes

$$
\begin{aligned}
v_{\tau}^{*}-\nu P \Delta v^{*}+\mathbb{P}\left(v^{*} \cdot \nabla v^{*}\right) & =\mathbb{P} f^{\varepsilon}, \\
\operatorname{div} v^{*} & =0, \\
v^{*}(0, y) & =u_{0}^{\varepsilon}(y),
\end{aligned}
$$

toujours dans le cas d'une force nulle.

Avant d'énoncer et de démontrer les résultats d'existence de solutions globales en dimension trois d'espace, nous décrivons une partie des résultats d'existence que nous avons obtenus en dimension deux d'espace ( [16]). Dans [16], dans le cas $n=2$, nous améliorons les résultats de [3] dans deux directions. D'une part, nous considérons des données initiales dans $H^{1}\left(\mathbb{R}^{2}\right)^{2} \times L^{2}(\mathbb{R})^{2}$ seulement (et même dans des espaces moins réguliers), et, d'autre part, nous comparons la solution $\left(u^{\varepsilon}, u_{\tau}^{\varepsilon}\right)$ à $\left(v^{*}, v_{\tau}^{*}\right)$ dans $H^{1}\left(\mathbb{R}^{2}\right)^{2} \times L^{2}\left(\mathbb{R}^{2}\right)^{2}$, où $v^{*}$ est la solution de (0.3). En particulier, les théorèmes démontrés dans [16] contiennent les résultats suivants. Dans la suite, on notera souvent $L^{p}\left((0,+\infty), L^{q}\left(\mathbb{R}^{n}\right)^{n}\right)$ (respectivement $L^{p}\left((0,+\infty), H^{s}\left(\mathbb{R}^{n}\right)^{n}\right)$ de manière abrégée par $L^{p}\left(L^{q}\right)$ (respectivement $\left.L^{p}\left(H^{s}\right)\right)$.

Théorème 0.1. (Cas $n=2$ ). Soit $\varepsilon_{0}>0$ donné. Il existe deux constantes strictement positives $K_{0}$, $K_{1}$ telles que, si $0<\varepsilon \leq \varepsilon_{0}$ et si les vecteurs à divergence nulle $u_{0}^{\varepsilon} \in H^{1}\left(\mathbb{R}^{2}\right)^{2}, u_{1}^{\varepsilon} \in L^{2}\left(\mathbb{R}^{2}\right)^{2}$ et la force $f^{\varepsilon} \in$ $L^{1}\left((0,+\infty), L^{2}\left(\mathbb{R}^{2}\right)^{2}\right) \cap L^{2}\left((0,+\infty), L^{2}\left(\mathbb{R}^{2}\right)^{2}\right)$ satisfont aux conditions

$$
\begin{aligned}
& \varepsilon\left\|u_{1}^{\varepsilon}\right\|_{L^{2}}+\varepsilon^{\frac{1}{2}}\left\|\nabla u_{0}^{\varepsilon}\right\|_{L^{2}}+\varepsilon^{\frac{1}{2}}\left\|f^{\varepsilon}\right\|_{L^{2}\left(L^{2}\right)} \leq K_{0} \\
& \varepsilon^{\frac{1}{4}}\left(\varepsilon^{\frac{1}{2}}\left\|u_{1}^{\varepsilon}\right\|_{L^{2}}+\left\|\nabla u_{0}^{\varepsilon}\right\|_{L^{2}}+\left\|f^{\varepsilon}\right\|_{L^{2}\left(L^{2}\right)}\right)^{\frac{1}{2}}\left(\left\|u_{0}^{\varepsilon}\right\|_{L^{2}}+\left\|f^{\varepsilon}\right\|_{L^{1}\left(L^{2}\right)}\right)^{\frac{1}{2}} \leq K_{1},
\end{aligned}
$$

l'équation (0.2) admet une (unique) solution intégrale globale $\left(u^{\varepsilon}, u_{\tau}^{\varepsilon}\right)$ dans $C^{0}\left([0,+\infty), H^{1}\left(\mathbb{R}^{2}\right)^{2} \times L^{2}\left(\mathbb{R}^{2}\right)^{2}\right)$. En outre, il existe une constante positive $C_{0}$ telle que, pour tout $\tau \geq 0$,

$$
\begin{aligned}
& \left\|u^{\varepsilon}(\tau)\right\|_{L^{2}}+\varepsilon\left\|u_{\tau}^{\varepsilon}(\tau)\right\|_{L^{2}}+\varepsilon^{\frac{1}{2}}\left\|\nabla u^{\varepsilon}(\tau)\right\|_{L^{2}} \\
& \quad \leq C_{0}\left(\left\|u_{0}^{\varepsilon}\right\|_{L^{2}}+\varepsilon\left\|u_{1}^{\varepsilon}\right\|_{L^{2}}+\varepsilon^{\frac{1}{2}}\left\|\nabla u_{0}^{\varepsilon}\right\|_{L^{2}}+\left\|f^{\varepsilon}\right\|_{L^{1}\left(L^{2}\right)}+\varepsilon^{\frac{1}{2}}\left\|f^{\varepsilon}\right\|_{L^{2}\left(L^{2}\right)}\right) .
\end{aligned}
$$


Le théorème 0.1 se démontre en faisant une décomposition en hautes et basses fréquences et en utilisant, à la fois, des estimations d'énergie et des inégalités de Strichartz.

Le résultat suivant est une conséquence directe du théorème 0.1 .

Corollaire 0.2. (Cas $n=2$ ) Pour tout $R>0$, il existe un nombre réel strictement positif $\varepsilon_{1}=\varepsilon_{1}(R)$ tel que, pour tout $0<\varepsilon \leq \varepsilon_{1}$, pour tout couple de vecteurs à divergence nulle $\left(u_{0}^{\varepsilon}, u_{1}^{\varepsilon}\right) \in H^{1}\left(\mathbb{R}^{2}\right)^{2} \times L^{2}\left(\mathbb{R}^{2}\right)^{2}$ et tout $f^{\varepsilon} \in L^{1}\left((0,+\infty), L^{2}\left(\mathbb{R}^{2}\right)^{2}\right) \cap L^{2}\left((0,+\infty), L^{2}\left(\mathbb{R}^{2}\right)^{2}\right)$ satisfaisant à la condition

$$
\left\|u_{0}^{\varepsilon}\right\|_{L^{2}}+\left\|u_{1}^{\varepsilon}\right\|_{L^{2}}+\left\|\nabla u_{0}^{\varepsilon}\right\|_{L^{2}}+\left\|f^{\varepsilon}\right\|_{L^{2}\left(L^{2}\right)}+\left\|f^{\varepsilon}\right\|_{L^{1}\left(L^{2}\right)} \leq R
$$

l'équation (0.1) admet une (unique) solution intégrale globale $\left(u^{\varepsilon}, u_{\tau}^{\varepsilon}\right)$ dans $C^{0}\left([0,+\infty), H^{1}\left(\mathbb{R}^{2}\right)^{2} \times L^{2}\left(\mathbb{R}^{2}\right)^{2}\right)$.

Quand $\varepsilon$ tend vers zéro, la limite formelle du système d'équations (0.1) (respectivement (0.2)) est le système des équations de Navier-Stokes (respectivement (0.3)). Dans le théorème suivant, on montre que cette limite n'est pas seulement formelle. Plus précisément, on montre que, sur tout intervalle de temps fini, on peut comparer la solution $\left(u^{\varepsilon}(\tau), u_{\tau}^{\varepsilon}(\tau)\right)$ de $(0.3)$ à $\left(v^{*}(\tau), v_{\tau}^{*}(\tau)\right)$ où $v^{*}(\tau)$ est la solution des équations $(0.3)$ avec donnée initiale $\left.u^{\varepsilon}(0)\right)$.

On rappelle que, pour tout $T>0$, pour tout vecteur $u_{0}^{\varepsilon} \in L^{2}\left(\mathbb{R}^{2}\right)^{2}$ à divergence nulle et toute force $f^{\varepsilon} \in$ $L^{2}\left((0, T) ; L^{2}\left(\mathbb{R}^{2}\right)^{2}\right)$, il existe une unique solution $\left.v^{*}(\tau) \in C^{0}\left((0, T) ; L^{2}\left(\mathbb{R}^{2}\right)^{2}\right)\right) \cap L_{l o c}^{2}\left((0, T) ; H^{1}\left(\mathbb{R}^{2}\right)^{2}\right)$ de $(0.3)$, de donnée initiale $v^{*}(0)=u_{0}^{\varepsilon}$. On notera cette solution $v^{*}(\tau)=S(\tau) u_{0}^{\varepsilon}$.

Soit $\beta>0$ fixé.

Théorème 0.3. (Cas $n=2$ ). Pour tous nombres strictement positifs $R$ et $T$, il existe un nombre strictement positif $\varepsilon_{1}=\varepsilon_{1}(R, T)$ tel que, pour $0<\varepsilon \leq \varepsilon_{1}$, si le couple de vecteurs à divergence nulle $\left(u_{0}^{\varepsilon}, u_{1}^{\varepsilon}\right) \in H^{1}\left(\mathbb{R}^{2}\right)^{2} \times$ $L^{2}\left(\mathbb{R}^{2}\right)^{2}$ et la force $f^{\varepsilon} \in L^{1}\left((0,+\infty), L^{2}\left(\mathbb{R}^{2}\right)^{2}\right) \cap L^{2}\left((0,+\infty), L^{2}\left(\mathbb{R}^{2}\right)^{2}\right)$ satisfont à la condition

$$
\left\|u_{0}^{\varepsilon}\right\|_{H^{1}}+\sqrt{\varepsilon}\left\|u_{1}^{\varepsilon}\right\|_{L^{2}}+\left\|f^{\varepsilon}\right\|_{L^{2}\left((0, T), L^{2}\right)}+\left\|((-\Delta+\beta I))^{-1 / 2} f^{\varepsilon}(0, .)\right\|_{L^{2}} \leq R,
$$

le système (0.2) admet une (unique) solution intégrale $\left(u^{\varepsilon}(\tau), u_{\tau}^{\varepsilon}(\tau)\right)$ dans $C^{0}\left([0,+\infty), H^{1}\left(\mathbb{R}^{2}\right)^{2} \times L^{2}\left(\mathbb{R}^{2}\right)^{2}\right)$, et on a, pour $0 \leq \tau \leq T$,

$$
\sqrt{\varepsilon}\left\|\frac{d}{d \tau} \tau\left(u^{\varepsilon}(\tau)-S(\tau) u_{0}^{\varepsilon}\right)\right\|_{L^{2}}+\left\|\tau\left(u^{\varepsilon}(\tau)-S(\tau) u_{0}^{\varepsilon}\right)\right\|_{H^{1}} \leq \varepsilon \exp K(T, R),
$$

où $K(R, T)$ est une constante positive ne dépendant que de $R$ et de $T$.

La démonstration du théorème 0.3 de comparaison est analogue à celle préconisée dans [18] (voir aussi [14]), mais elle est un peu plus longue à cause de l'utilisation des inégalités de Strichartz. En particulier, on utilise la décomposition du système (0.2) en deux systèmes associés, indiquée dans [18].

Dans cet article-ci, nous démontrons l'existence globale de solutions en dimension trois d'espace. Comme pour les équations de Navier-Stokes en dimension trois, nous n'obtenons l'existence globale de solutions que sous une hypothèse de petitesse sur les données initiales et le terme de force. Dans l'énoncé du théorème d'existence global de solutions, nous utiliserons des espaces de Sobolev d'indice fractionnaire dont nous rappelons rapidement la définition.

Pour tout $s \in \mathbb{R}$, on introduit l'espace (non-homogène) sur $\mathbb{R}^{n}$ :

$$
H^{s}\left(\mathbb{R}^{n}\right)=\left\{u \in \mathcal{S}^{\prime}\left(\mathbb{R}^{n}\right) \mid \hat{u} \in L_{l o c}^{2}\left(\mathbb{R}^{n}\right) \text { et } \int_{\mathbb{R}^{n}}\left(1+|\xi|^{2}\right)^{s}|\hat{u}(\xi)|^{2} d \xi<+\infty\right\}
$$

qu'on munit de la norme sur $H^{s}\left(\mathbb{R}^{n}\right)$, donnée par

$$
\|u\|_{H^{s}}^{2}=\int_{\mathbb{R}^{n}}\left(1+|\xi|^{2}\right)^{s}|\hat{u}(\xi)|^{2} d \xi
$$


où $\hat{u}$ désigne la transformée de Fourier de $u$. On note $(\cdot, \cdot)_{H^{s}}$ le produit scalaire correspondant. Le produit scalaire dans $L^{2}\left(\mathbb{R}^{n}\right)$ sera simplement noté $(\cdot, \cdot)$. On définit aussi l'espace homogène correspondant

$$
\dot{H}^{s}\left(\mathbb{R}^{n}\right)=\left\{u \in \mathcal{S}^{\prime}\left(\mathbb{R}^{n}\right) \mid \hat{u} \in L_{l o c}^{1}\left(\mathbb{R}^{n}\right) \text { et } \int_{\mathbb{R}^{n}}|\xi|^{2 s}|\hat{u}(\xi)|^{2} d \xi<+\infty\right\}
$$

et on pose

Pour $u$ et $v$ dans $\dot{H}^{s}\left(\mathbb{R}^{n}\right)$, on pose

$$
\|u\|_{\dot{H}^{s}}^{2} \equiv\|u\|_{s}^{2} \equiv \int_{\mathbb{R}^{n}}|\xi|^{2 s}|\hat{u}(\xi)|^{2} d \xi
$$

$$
(u, v)_{s}=\int_{\mathbb{R}^{n}}|\xi|^{2 s} \hat{u}(\xi) \cdot \overline{\hat{v}(\xi)} d \xi .
$$

Dans cet article, nous démontrons les résultats d'existence globale suivants.

Théorème 0.4. (Cas $n=3$ ). Soit $\varepsilon_{0}>0$ fixé. Soit $d>0$ et $0<\delta \leq \frac{1}{2}$ deux nombres réels donnés. Il existe deux constantes strictement positives $K_{0}$ et $K_{1}$ telles que si, $0<\varepsilon \leq \varepsilon_{0}$ et si le couple de vecteurs à divergence nulle $\left(u_{0}^{\varepsilon}, u_{1}^{\varepsilon}\right) \in H^{1+\delta}\left(\mathbb{R}^{3}\right)^{3} \times H^{\delta}\left(\mathbb{R}^{3}\right)^{3}$ et la force $f^{\varepsilon} \in L^{1}\left((0,+\infty), L^{2}\left(\mathbb{R}^{3}\right)^{3}\right) \cap L^{2}\left((0,+\infty), H^{\delta}\left(\mathbb{R}^{3}\right)^{3}\right) \cap$ $\left.L^{p}\left((0,+\infty), \dot{H}^{\frac{p-1}{p}+d}\left(\mathbb{R}^{3}\right)^{3}\right)\right)$, où $1 \leq p \leq 2$ et où $\frac{3}{4}-\frac{1}{p}+\frac{p-1}{2 p}+\frac{d}{2}>0$, satisfont aux conditions

$$
\begin{aligned}
\varepsilon^{\frac{3}{4}}\left(\left\|u_{1}^{\varepsilon}\right\|_{L^{2}}+\right. & \left.\varepsilon^{\frac{\delta}{2}}\left\|u_{1}^{\varepsilon}\right\|_{\dot{H}^{\delta}}\right)+\varepsilon^{\frac{1}{4}}\left(\left\|\nabla u_{0}^{\varepsilon}\right\|_{L^{2}}+\varepsilon^{\frac{\delta}{2}}\left\|\nabla u_{0}^{\varepsilon}\right\|_{\dot{H}^{\delta}}\right) \\
& +\varepsilon^{\frac{1}{4}}\left(\left\|f^{\varepsilon}\right\|_{L^{2}\left(L^{2}\right)}+\varepsilon^{\frac{\delta}{2}}\left\|f^{\varepsilon}\right\|_{L^{2}\left(\dot{H}^{\delta}\right)}\right)+\varepsilon^{\frac{3}{4}+\frac{p-3}{2 p}+\frac{d}{2}}\left\|f^{\varepsilon}\right\|_{L^{p}\left(\dot{H}^{\frac{p-1}{p}+d}\right)} \leq K_{0} \\
{\left[\varepsilon ^ { \frac { 3 } { 4 } } \left(\left\|u_{1}^{\varepsilon}\right\|_{L^{2}}\right.\right.} & \left.\left.+\varepsilon^{\frac{\delta}{2}}\left\|u_{1}^{\varepsilon}\right\|_{\dot{H}^{\delta}}\right)+\varepsilon^{\frac{1}{4}}\left(\left\|\nabla u_{0}^{\varepsilon}\right\|_{L^{2}}+\varepsilon^{\frac{\delta}{2}}\left\|\nabla u_{0}^{\varepsilon}\right\|_{\dot{H}^{\delta}}\right)+\varepsilon^{\frac{1}{4}}\left(\left\|f^{\varepsilon}\right\|_{L^{2}\left(L^{2}\right)}+\varepsilon^{\frac{\delta}{2}}\left\|f^{\varepsilon}\right\|_{L^{2}\left(\dot{H}^{\delta}\right)}\right)\right]^{\frac{1}{2}} \\
& \times\left[\varepsilon^{-\frac{1}{4}}\left\|u_{0}^{\varepsilon}\right\|_{L^{2}}+\varepsilon^{\frac{3}{4}}\left\|u_{1}^{\varepsilon}\right\|_{L^{2}}+\varepsilon^{\frac{1}{4}}\left\|\nabla u_{0}^{\varepsilon}\right\|_{L^{2}}+\varepsilon^{\frac{1}{4}}\left\|f^{\varepsilon}\right\|_{L^{2}\left(L^{2}\right)}+\varepsilon^{-\frac{1}{4}}\left\|f^{\varepsilon}\right\|_{L^{1}\left(L^{2}\right)}\right]^{\frac{1}{2}} \leq K_{1},
\end{aligned}
$$

l'équation (0.2) admet une (unique) solution intégrale globale $\left(u^{\varepsilon}, u_{\tau}^{\varepsilon}\right)$ dans $C^{0}\left([0,+\infty), H^{1+\delta}\left(\mathbb{R}^{3}\right)^{3} \times H^{\delta}\left(\mathbb{R}^{3}\right)^{3}\right)$. En outre, il existe une constante strictement positive $C_{0}$ telle que, pour tout $t \geq 0$,

$$
\begin{aligned}
& \varepsilon^{\frac{3}{4}}\left(\left\|u_{t}^{\varepsilon}(t)\right\|_{L^{2}}+\varepsilon^{\frac{\delta}{2}}\left\|u_{t}^{\varepsilon}(t)\right\|_{\dot{H}^{\delta}}\right)+\varepsilon^{\frac{1}{4}}\left(\left\|\nabla u^{\varepsilon}(t)\right\|_{L^{2}}+\varepsilon^{\frac{\delta}{2}}\left\|\nabla u^{\varepsilon}(t)\right\|_{\dot{H}^{\delta}}\right) \\
& \quad \leq C_{0}\left[\varepsilon^{\frac{3}{4}}\left(\left\|u_{1}^{\varepsilon}\right\|_{L^{2}}+\varepsilon^{\frac{\delta}{2}}\left\|u_{1}^{\varepsilon}\right\|_{\dot{H}^{\delta}}\right)+\varepsilon^{\frac{1}{4}}\left(\left\|\nabla u_{0}^{\varepsilon}\right\|_{L^{2}}+\varepsilon^{\frac{\delta}{2}}\left\|\nabla u_{0}^{\varepsilon}\right\|_{\dot{H}^{\delta}}\right)+\varepsilon^{\frac{1}{4}}\left(\left\|f^{\varepsilon}\right\|_{L^{2}\left(L^{2}\right)}+\varepsilon^{\frac{\delta}{2}}\left\|f^{\varepsilon}\right\|_{L^{2}\left(\dot{H}^{\delta}\right)}\right)\right],
\end{aligned}
$$

et

$$
\begin{gathered}
\varepsilon^{-\frac{1}{4}}\left\|u^{\varepsilon}(t)\right\|_{L^{2}} \leq C_{0}\left[\varepsilon^{\frac{3}{4}}\left(\left\|u_{1}^{\varepsilon}\right\|_{L^{2}}+\varepsilon^{\frac{\delta}{2}}\left\|u_{1}^{\varepsilon}\right\|_{\dot{H}^{\delta}}\right)+\varepsilon^{\frac{1}{4}}\left(\left\|\nabla u_{0}^{\varepsilon}\right\|_{L^{2}}+\varepsilon^{\frac{\delta}{2}}\left\|\nabla u_{0}^{\varepsilon}\right\|_{\dot{H}^{\delta}}\right)+\varepsilon^{\frac{1}{4}}\left(\left\|f^{\varepsilon}\right\|_{L^{2}\left(L^{2}\right)}+\varepsilon^{\frac{\delta}{2}}\left\|f^{\varepsilon}\right\|_{L^{2}\left(\dot{H}^{\delta}\right)}\right)\right. \\
\left.+\varepsilon^{-\frac{1}{4}}\left\|u_{0}^{\varepsilon}\right\|_{L^{2}}+\varepsilon^{-\frac{1}{4}}\left\|f^{\varepsilon}\right\|_{L^{1}\left(L^{2}\right)}\right]
\end{gathered}
$$

On déduit immédiatement de ce théorème le corollaire suivant.

Corollaire 0.5. (Cas $n=3$ ) Pour tous nombres réels $0 \leq \delta \leq \frac{1}{2}$ et $d>0$, il existe une constante strictement positive $K_{2}=K_{2}(\delta, d)$, et, pour tout nombre réel $R>0$, il existe un nombre strictement positif $\varepsilon_{1}=\varepsilon_{1}(\delta, d, R)$, tels que, pour tout couple de vecteurs à divergence nulle $\left(u_{0}^{\varepsilon}, u_{1}^{\varepsilon}\right) \in H^{\delta+1}\left(\mathbb{R}^{3}\right)^{3} \times H^{\delta}\left(\mathbb{R}^{3}\right)^{3}$ et toute force $f^{\varepsilon} \in$ $L^{1}\left((0,+\infty), L^{2}\left(\mathbb{R}^{3}\right)^{3}\right) \cap L^{2}\left((0,+\infty), H^{\delta}\left(\mathbb{R}^{3}\right)^{3}\right) \cap L^{p}\left((0,+\infty), \dot{H}^{\frac{p-1}{p}+d}\left(\mathbb{R}^{3}\right)^{3}\right)$ (où $1 \leq p \leq 2$ et ò̀ $\frac{3}{4}-\frac{1}{p}+\frac{p-1}{2 p}+\frac{d}{2}>$ $0)$, satisfaisant aux conditions

$$
\begin{aligned}
&\left(\left\|u_{0}^{\varepsilon}\right\|_{L^{2}}+\left\|f^{\varepsilon}\right\|_{L^{1}\left(L^{2}\right)}\right) \times\left(\left\|\nabla u_{0}^{\varepsilon}\right\|_{L^{2}}+\left\|f^{\varepsilon}\right\|_{L^{2}\left(L^{2}\right)}\right) \leq K_{2} \\
&\left\|u_{0}^{\varepsilon}\right\|_{L^{2}}+\left\|\nabla u_{0}^{\varepsilon}\right\|_{H^{\delta}}+\left\|u_{1}^{\varepsilon}\right\|_{H^{\delta}}+\left\|f^{\varepsilon}\right\|_{L^{2}\left(H^{\delta}\right)}+\left\|f^{\varepsilon}\right\|_{L^{1}\left(L^{2}\right)}+\left\|f^{\varepsilon}\right\|_{L^{p}\left(\dot{H}^{\frac{p-1}{p}+d}\right)} \leq R
\end{aligned}
$$


l'équation (0.2) admet une (unique) solution intégrale globale $\left(u^{\varepsilon}, u_{\tau}^{\varepsilon}\right)$ dans $C^{0}\left([0,+\infty), H^{1+\delta}\left(\mathbb{R}^{3}\right)^{3} \times H^{\delta}\left(\mathbb{R}^{3}\right)^{3}\right)$.

On pourrait démontrer un analogue du théorème 0.3 permettant de comparer les solutions du système $(0.2)$ aux solutions du système des équations de Navier-Stokes (0.3) sur tout intervalle de temps fini. Comme la démonstration est vraiment semblable à celle faite dans le cas de la dimension deux, nous renvoyons le lecteur à l'article [16].

Dans un article à venir, nous étudierons aussi le comportement asymptotique en temps des solutions des équations (0.2), en dimension deux d'espace, quand $\varepsilon>0$ est suffisamment petit. En particulier, nous le comparerons à celui des équations de Navier-Stokes correspondantes.

Le plan de cet article est le suivant. Dans un premier paragraphe, nous introduisons tous les outils nécessaires pour démontrer le théorème 0.4 . Nous faisons d'abord le changement de variables qui ramène les équations $(0.2)$ à des équations indépendantes de $\varepsilon$, mais où la donnée initiale et la force dépendent de $\varepsilon$. Ensuite, nous faisons une décomposition en basses et hautes fréqences des solutions et enfin, nous rappelons des inégalités de type Strichartz. Au paragraphe deux, nous démontrons le théorème 0.4 en plusieurs étapes.

\section{Préliminaires}

\subsection{Changements de variables}

Pour démontrer des inégalités de type Strichartz ainsi que l'existence globale de solutions, il vaut mieux travailler avec des équations dont les coefficients sont indépendants de $\varepsilon$. Pour cette raison, on fait le changement d'échelle suivant :

$$
\begin{aligned}
& u^{\varepsilon}(\tau, y)=\frac{1}{\sqrt{\varepsilon}} u\left(\frac{\tau}{\varepsilon}, \frac{y}{\sqrt{\varepsilon}}\right), f^{\varepsilon}(\tau, y)=\frac{1}{\varepsilon \sqrt{\varepsilon}} f\left(\frac{\tau}{\varepsilon}, \frac{y}{\sqrt{\varepsilon}}\right) \\
& p^{\varepsilon}(\tau, y)=\frac{1}{\varepsilon} p\left(\frac{\tau}{\varepsilon}, \frac{y}{\sqrt{\varepsilon}}\right)
\end{aligned}
$$

On pose :

$$
t=\frac{\tau}{\varepsilon}, \quad x=\frac{y}{\sqrt{\varepsilon}} .
$$

Ce changement d'échelle transforme les équations

$$
\begin{aligned}
\varepsilon u_{\tau \tau}^{\varepsilon}+u_{\tau}^{\varepsilon}-\nu \Delta u^{\varepsilon} & =-u^{\varepsilon} \cdot \nabla u^{\varepsilon}-\nabla p^{\varepsilon}+f^{\varepsilon} \\
\operatorname{div} u^{\varepsilon} & =0, \\
\left(u^{\varepsilon}, u_{\tau}^{\varepsilon}\right)(0, y) & =\left(u_{0}^{\varepsilon}(y), u_{1}^{\varepsilon}(y)\right),
\end{aligned}
$$

en le système ci-dessous, où $\varepsilon$ transparaît seulement dans les conditions initiales et dans le terme de force :

$$
\begin{aligned}
u_{t t}+u_{t}-\nu \Delta u & =-u \cdot \nabla u-\nabla p+f \\
\operatorname{div} u & =0 \\
\left(u, u_{t}\right)(0, x) & =\left(\sqrt{\varepsilon} u_{0}^{\varepsilon}(\sqrt{\varepsilon} x), \varepsilon^{3 / 2} u_{1}^{\varepsilon}(\sqrt{\varepsilon} x)\right) \equiv\left(u_{0}, u_{1}\right) .
\end{aligned}
$$

Un calcul élémentaire et rapide montre que, par ce changement d'échelle, on a les égalités de normes suivantes :

$$
\begin{gathered}
\|u\|_{\dot{H}_{x}^{s}}=\varepsilon^{\frac{s}{2}-\frac{1}{4}}\left\|u^{\varepsilon}\right\|_{\dot{H}_{y}^{s}}, \quad \forall s \geq 0, \\
\|u\|_{L_{x}^{p}}=\varepsilon^{-\frac{3}{2 p}+\frac{1}{2}}\left\|u^{\varepsilon}\right\|_{L_{y}^{p}}, \quad \forall p \geq 1,
\end{gathered}
$$


et, en particulier,

$$
\|u\|_{L_{x}^{2}}=\varepsilon^{-\frac{1}{4}}\left\|u^{\varepsilon}\right\|_{L_{y}^{2}},\|\nabla u\|_{L_{x}^{2}}=\varepsilon^{\frac{1}{4}}\left\|\nabla u^{\varepsilon}\right\|_{L_{y}^{2}},\left\|u_{t}\right\|_{L_{x}^{2}}=\varepsilon^{\frac{3}{4}}\left\|u_{\tau}^{\varepsilon}\right\|_{L_{y}^{2}}, .
$$

Un calcul tout aussi élémentaire montre que, pour $p \geq 1$, on a les égalités suivantes :

$$
\|f\|_{L^{p}\left(\dot{H}_{x}^{s}\right)}=\varepsilon^{\frac{3}{4}+\frac{s}{2}-\frac{1}{p}}\left\|f^{\varepsilon}\right\|_{L^{p}\left(\dot{H}_{y}^{s}\right)}, \quad \forall p \geq 1, \quad \forall s \geq 0 .
$$

\subsection{Décomposition en basses fréquences et hautes fréquences}

Parmi les outils de base utilisés dans la démonstration du théorème 0.4 figure la théorie de Littlewood-Paley. Nous rappelons rapidement ici la définition et les propriétés des décompositions dyadiques.

On introduit une fonction paire $\chi^{*} \in C_{0}^{\infty}(\mathbb{R})$ telle que le support de $\chi^{*}$ soit contenu dans la boule $B_{\mathbb{R}}\left(0, \frac{4}{3}\right)$ de centre 0 et de rayon $\frac{4}{3}$ dans $\mathbb{R}$ et que $\chi^{*}$ soit égale à 1 dans un voisinage de la boule $B_{\mathbb{R}}\left(0, \frac{3}{4}\right)$. Si on pose

$$
\varphi^{*}(\xi)=\chi^{*}\left(\frac{\xi}{2}\right)-\chi^{*}(\xi),
$$

on voit que le support de $\varphi^{*}$ est contenu dans la couronne $\mathcal{C}_{\mathbb{R}}\left(\frac{3}{4}, \frac{8}{3}\right) \equiv\left\{\xi \in \mathbb{R}\left|\frac{3}{4} \leq\right| \xi \mid \leq \frac{8}{3}\right\}$ et que $\varphi^{*}$ est identiquement égale à 1 sur la couronne $\mathcal{C}_{\mathbb{R}}\left(\frac{4}{3}, \frac{3}{2}\right)$.

Pour $j \in \mathbb{Z}$, on pose

$$
\varphi_{j}^{*}(\xi)=\varphi^{*}\left(\frac{\xi}{2^{j}}\right) .
$$

Le support de $\varphi_{j}^{*}$ est contenu dans la couronne $2^{j} \mathcal{C}_{\mathbb{R}}\left(\frac{3}{4}, \frac{8}{3}\right)$ et $\varphi_{j}^{*}$ est identiquement égale à 1 sur la couronne $2^{j} \mathcal{C}_{\mathbb{R}}\left(\frac{4}{3}, \frac{3}{2}\right)$. On remarque que

$$
\chi^{*}(\xi)+\sum_{j=0}^{\infty} \varphi_{j}^{*}(\xi)=1, \quad \forall \xi \in \mathbb{R}
$$

et que

$$
\operatorname{supp}\left(\varphi_{p}^{*}\right) \cap \operatorname{supp}\left(\varphi_{q}^{*}\right)=\emptyset, \quad \forall p, q|p-q| \geq 2 .
$$

On définit ensuite les fonctions $\varphi$ et $\chi$ sur $\mathbb{R}^{3}$ en posant

$$
\varphi(x)=\varphi^{*}(|x|), \quad \chi(x)=\chi^{*}(|x|), \quad \forall x \in \mathbb{R}^{3} .
$$

Si $u$ est un élément de $\mathcal{S}^{\prime}\left(\mathbb{R}^{3}\right)$, on note $\mathcal{F}(u)$ la transformée de Fourier de $u$ et $\mathcal{F}^{-1}(u)$ la transformée de Fourier inverse de $u$. Maintenant, on est en mesure de définir les opérateurs de troncature en fréquences suivantes, pour tout $u \in \mathcal{S}^{\prime}\left(\mathbb{R}^{3}\right)$,

$$
\begin{aligned}
\Delta_{j} u & =\mathcal{F}^{-1}\left(\varphi_{j}(\xi) \mathcal{F}(u)(\xi)\right), \quad \forall j \in \mathbb{N} \\
\Delta_{-1} u & =\mathcal{F}^{-1}(\chi(\xi) \mathcal{F}(u)(\xi)), \\
\Delta_{j} u & =0, \quad \forall j, j \leq-2 . \\
S_{q} u & =\sum_{j \leq q-1} \Delta_{j} u, \quad \forall q, q \geq 1 .
\end{aligned}
$$

L'égalité (1.7) et la définition (1.8) entrainnent que tout élément $u \in \mathcal{S}^{\prime}\left(\mathbb{R}^{3}\right)$ s'écrit

$$
u=\sum_{j \geq-1} \Delta_{j} u .
$$


Dans la suite, on aura besoin du lemme de Bernstein suivant (pour une démonstration, voir [6] par exemple). Soit $\mathcal{C}=\mathcal{C}\left(r_{1}, r_{2}\right)$ une couronne de petit rayon $r_{1}>0$ et de grand rayon $r_{2}>r_{1}$.

Lemme 1.1. (Lemme de Bernstein) Il existe une constante $C_{0}$ strictement positive telle que, pour tout élément $u$ de $\mathcal{S}^{\prime}\left(\mathbb{R}^{3}\right)$ ayant le support de $\mathcal{F} u$ contenu dans la couronne $2^{q} \mathcal{C}$, où $q \in \mathbb{Z}$, on a les estimations suivantes,

$$
2^{q k} C_{0}^{-k}\|u\|_{L^{p}} \leq \sup _{|\alpha|=k}\left\|\partial^{\alpha} u\right\|_{L^{p}} \leq 2^{q k} C_{0}^{k}\|u\|_{L^{p}}, \quad \forall k \geq 0, \quad \forall p \geq 1
$$

et

$$
\|u\|_{L^{p^{\prime}}} \leq C_{0} 2^{3 q\left(\frac{1}{p}-\frac{1}{p^{\prime}}\right)}\|u\|_{L^{p}}, \quad \forall p^{\prime} \geq p \geq 1 .
$$

De même, pour tout élément $u$ de $\mathcal{S}^{\prime}\left(\mathbb{R}^{3}\right)$ ayant le support de $\mathcal{F} u$ contenu dans $\cup_{-1 \leq j \leq q} 2^{j} \mathcal{C}$, où $q \in \mathbb{N}$, on a les estimations suivantes,

$$
\begin{array}{r}
\sup _{|\alpha|=k}\left\|\partial^{\alpha} u\right\|_{L^{p}} \leq 2^{q k} C_{0}^{k}\|u\|_{L^{p}}, \quad \forall k \geq 0, \quad \forall p \geq 1 \\
\|u\|_{L^{p^{\prime}}} \leq C_{0} 2^{3 q\left(\frac{1}{p}-\frac{1}{p^{\prime}}\right)}\|u\|_{L^{p}}, \quad \forall p^{\prime} \geq p \geq 1 .
\end{array}
$$

La décomposition dyadique introduite ci-dessus nous permet de donner une définition alternative de l'espace de Sobolev $H^{s}\left(\mathbb{R}^{3}\right)$. En effet, du lemme de Bernstein, on déduit qu'il existe une constante $C_{1}$ strictement positive telle que, pour tout $s \geq 0$,

$$
C_{1}^{-s-1}\|u\|_{H^{s}}^{2} \leq \sum_{j \geq-1} 2^{2 j s}\left\|\Delta_{j} u\right\|_{L^{2}}^{2} \leq C_{1}^{s+1}\|u\|_{H^{s}}^{2} .
$$

On peut facilement définir les espaces de Besov $B_{p, r}^{s}\left(\mathbb{R}^{3}\right)$, où $s, p$ et $r$ sont des nombres réels, $p \geq 1, r \geq 1$, grâce aux décompositions dyadiques,

$$
B_{p, r}^{s}\left(\mathbb{R}^{3}\right)=\left\{w \in \mathcal{S}^{\prime}\left(\mathbb{R}^{3}\right) \mid\|w\|_{B_{p, r}^{s}} \equiv\left(\sum_{j \geq-1} 2^{j r s}\left\|\Delta_{j} w\right\|_{L^{p}}^{r}\right)^{\frac{1}{r}}<+\infty\right\}
$$

Remarquons que, dans la démonstration des inégalités de Strichartz, on utilise l'inclusion continue suivante

$$
B_{p, 1}^{0}\left(\mathbb{R}^{3}\right) \hookrightarrow L^{p}\left(\mathbb{R}^{3}\right), \quad \forall p \geq 1
$$

Dans la preuve du théorème 0.4 , nous aurons besoin d'estimations de $\|u\|_{L_{t}^{p}\left(L^{\infty}\right)}$, où $u$ est la solution du système (1.3), où $p \geq 1$ et où $L_{t}^{p}\left(L^{\infty}\right)=L^{p}\left((0, t),\left(L^{\infty}\left(\mathbb{R}^{3}\right)\right)^{3}\right)$. Dans ce but, nous allons décomposer $u$ comme suit

$$
u=\sum_{j=-1}^{j_{0}-1} \Delta_{j} u+\sum_{j=j_{0}}^{+\infty} \Delta_{j} u \equiv \tilde{S}_{0} u+\left(I-\tilde{S}_{0}\right) u,
$$

où $j_{0}$ est un nombre entier positif bien choisi.

Remarques 1.1. a) On note qu'il existe une constante $C_{2}$ strictement positive telle que, pour tout $s \geq 0$,

$$
\left\|\tilde{S}_{0} u\right\|_{s} \leq C_{2}\|u\|_{s}, \quad\left\|\left(I-\tilde{S}_{0}\right) u\right\|_{s} \leq C_{2}\|u\|_{s} .
$$

b) On remarque aussi que, si $u$ est un vecteur à divergence nulle, alors $\tilde{S}_{0} u$ et $\left(I-\tilde{S}_{0}\right) u$ sont aussi des vecteurs à divergence nulle.

c) Enfin, on remarque que $\tilde{S}_{0} u$ contient les basses fréquences et est aussi régulier que l'on veut tandis que $\left(I-\tilde{S}_{0}\right) u$ contient les hautes fréquences et a la même régularité que $u$. Au cours de la démonstration du théorème 0.4 , on 
estimera $\left\|\tilde{S}_{0} u\right\|_{L_{t}^{\infty}\left(L^{\infty}\right)}$ grâce à un argument de fonctionnelle d'énergie et au lemme de Bernstein. Au paragraphe suivant, on donnera une estimation de $\left\|\left(I-\tilde{S}_{0}\right) u\right\|_{L_{t}^{2}\left(L^{\infty}\right)}$ en utilisant des inégalités de Strichartz obtenues pour le système affine correspondant à (1.3). On précisera aussi le choix de $j_{0}$.

On termine ce paragraphe par un rappel des lois de produit ci-dessous, qui sont des variantes des estimations de produits classiques (voir [1] ou [6]).

Proposition 1.2. 1) Pour tout nombre réel $s \geq 0$, il existe une constante strictement positive $C_{3}=C_{3}(s)$ telle que, pour tout $u_{1}$ et tout $u_{2}$, où $u_{i} \in L^{\infty}\left(\mathbb{R}^{3}\right) \cap \dot{H}^{s+1}\left(\mathbb{R}^{3}\right)$, pour $i=1,2$, on a

$$
\left\|u_{1} \nabla u_{2}\right\|_{s} \leq C_{3}\left(\left\|u_{1}\right\|_{L^{\infty}}\left\|u_{2}\right\|_{s+1}+\left\|u_{2}\right\|_{L^{\infty}}\left\|u_{1}\right\|_{s+1}\right)
$$

2) Pour tous nombres réels $s_{1}$ et $s_{2}$, où $s_{1}<3 / 2, s_{2}<3 / 2, s_{1}+s_{2}>0$, si $u_{i} \in \dot{H}^{s_{i}}\left(\mathbb{R}^{3}\right)$ pour $i=1,2$, alors $u_{1} u_{2}$ appartient à l'espace $\dot{H}^{s_{1}+s_{2}-3 / 2}\left(\mathbb{R}^{3}\right)$ et on a l'inégalité

$$
\left\|u_{1} u_{2}\right\|_{s_{1}+s_{2}-3 / 2} \leq C_{3}^{*}\left(\left(s_{1}+s_{2}\right)^{-1 / 2}+\left(3 / 2-s_{1}\right)^{-1 / 2}+\left(3 / 2-s_{2}\right)^{-1 / 2}\right)\left\|u_{1}\right\|_{s_{1}}\left\|u_{2}\right\|_{s_{2}},
$$

où $C_{3}^{*}$ est une constante strictement positive indépendante de $u_{i}$ et de $s_{i}, i=1,2$. Si, en outre, le support de $\mathcal{F} u_{1}$ est contenu dans $\cup_{-1 \leq j \leq q} 2^{j} \mathcal{C}$, où $q \in \mathbb{N}$, si $u_{2}$ appartient à $\dot{H}^{s_{1}+s_{2}-3 / 2}\left(\mathbb{R}^{3}\right)$ et si $s_{1}+s_{2}-3 / 2 \geq 0$, on obtient l'estimation suivante

$$
\left\|u_{1} u_{2}\right\|_{s_{1}+s_{2}-3 / 2} \leq C_{3}^{*}\left(\left(s_{1}+s_{2}\right)^{-1 / 2}+\left(3 / 2-s_{2}\right)^{-1 / 2}\right)\left\|u_{1}\right\|_{s_{1}}\left\|u_{2}\right\|_{s_{2}}+C_{p, q}\left\|u_{1}\right\|_{L^{p}}\left\|u_{2}\right\|_{s_{1}+s_{2}-3 / 2},
$$

où $p>1$ est un nombre réel ou bien $p=+\infty$.

La démonstration de l'inégalité (1.18) est donnée dans [6] et [1] par exemple (voir aussi [17]). Comme les inégalités (1.17) et (1.19) ne figurent nulle part sous cette forme précise, nous donnons leur démonstration dans l'annexe A.

\subsection{Inégalités de Strichartz}

Dans ce paragraphe, nous énoncerons les inégalités de Strichartz pour le système affine associé au système (1.3). Si on applique le projecteur de Leray $\mathbb{P}$ aux équations (1.3), on obtient le système

$$
\begin{aligned}
u_{t t}+u_{t}-\nu \Delta u & =-\mathbb{P}(u \cdot \nabla u)+\mathbb{P} f, \\
\operatorname{div} u & =0 \\
\left(u, u_{t}\right)(0, x) & =\left(u_{0}, u_{1}\right) .
\end{aligned}
$$

Pour obtenir une estimation de $\|u\|_{L_{t}^{2}\left(L^{\infty}\right)}$ ou plutôt de $\left\|\left(I-\tilde{S}_{0}\right) u\right\|_{L_{t}^{2}\left(L^{\infty}\right)}$, on introduit le système linéaire non homogène suivant, associé au système (1.20),

$$
\begin{aligned}
U_{t t}+U_{t}-\nu \Delta U & =G, \\
\operatorname{div} U & =0, \\
\left(U, U_{t}\right)(0, x) & =\left(u_{0}, u_{1}\right),
\end{aligned}
$$

où $G$ est un terme de force. Et on démontre des inégalités de type Strichartz pour le système (1.21). Si on applique l'opérateur $\Delta_{j}$ aux équations (1.21), on obtient le système

$$
\begin{aligned}
\left(\Delta_{j} U\right)_{t t}+\left(\Delta_{j} U\right)_{t}-\nu \Delta_{j} U & =\Delta_{j} G \\
\operatorname{div} \Delta_{j} U & =0 \\
\left(\Delta_{j} U,\left(\Delta_{j} U\right)_{t}\right)(0, x) & =\left(\Delta_{j} u_{0}, \Delta_{j} u_{1}\right)
\end{aligned}
$$


On remarque que le support de $\Delta_{j} U$ est contenu dans la couronne $2^{j} \mathcal{C}$. Pour nous ramener à un support inclus dans la couronne fixe $\mathcal{C}$ pour tout $j \geq 0$, on pose

$$
U_{j}(\tau, y)=\Delta_{j} U\left(2^{-j} \tau, 2^{-j} y\right)
$$

Pour tout $j \geq 0, U_{j}$ satisfait à l'équation suivante:

$$
\begin{aligned}
\left(U_{j}\right)_{\tau \tau}+2^{-j}\left(U_{j}\right)_{\tau}-\nu \Delta U_{j} & =2^{-2 j} \Delta_{j} G\left(2^{-j} \tau, 2^{-j} y\right) \equiv 2^{-2 j} G_{j}(\tau, y) \\
\operatorname{div} U_{j} & =0 \\
\left(U_{j},\left(U_{j}\right)_{\tau}\right)(0, y) & =\left(\Delta_{j} u_{0}\left(2^{-j} y\right), 2^{-j} \Delta_{j} u_{1}\left(2^{-j} y\right)\right) \equiv\left(u_{0, j}, u_{1, j}\right)
\end{aligned}
$$

ou encore, si $\widehat{U}_{j} \equiv \mathcal{F} U_{j}$ désigne la transformée de Fourier de $U_{j}$,

$$
\begin{aligned}
\widehat{U}_{j, \tau \tau}+2^{-j} \widehat{U}_{j, \tau}+\nu|\xi|^{2} \widehat{U}_{j} & =2^{-2 j} \widehat{G}_{j}, \\
\left(\widehat{U}_{j}, \widehat{U}_{j, \tau}\right)(0, y) & =\left(\widehat{u}_{0, j}, \widehat{u}_{1, j}\right) .
\end{aligned}
$$

Par exemple, dans le cas où $G$ est nulle, la solution $U_{j}$ du système (1.24) s'écrit

$$
\widehat{U}_{j}(\tau, \xi)=\left(\lambda_{-}-\lambda_{+}\right)^{-1}\left(\left(\lambda_{-} \widehat{u}_{0, j}-\widehat{u}_{1, j}\right) e^{\lambda_{+} t}+\left(\widehat{u}_{1, j}-\lambda_{+} \widehat{u}_{0, j}\right) e^{\lambda_{-} t}\right)
$$

où

$$
\lambda_{ \pm}=\lambda_{ \pm}(\xi)=\frac{1}{2}\left(-2^{-j} \pm\left(\left(2^{-2 j}-4|\xi|^{2}\right)^{\frac{1}{2}}\right) .\right.
$$

Puisque le support de $U_{j}$ est inclus dans la couronne fixe $\mathcal{C}$, on remarque qu'il existe $j_{0}>0$ tel que, pour $j \geq j_{0}$, $4|\xi|^{2}-2^{-2 j}>0$ et donc

$$
\lambda_{ \pm}=\lambda_{ \pm}(\xi)=\frac{1}{2}\left(-2^{-j} \pm i \sqrt{4|\xi|^{2}-2^{-2 j}}\right) .
$$

Donc, quand $j \geq j_{0}$, nous sommes dans le cas hyperbolique et on pourra démontrer des inégalités de type Strichartz pour $\left(I-\tilde{S}_{0}\right) U \equiv \sum_{j=j_{0}}^{+\infty} \Delta_{j} U$. Dans le cas contraire où $j \geq 0$ est petit, nous sommes dans le régime parabolique.

Dans la suite, on fixe $j_{0}>0$ tel que, pour $j \geq j_{0}, 4|\xi|^{2}-2^{-2 j}>0$. Dans l'appendice de l'article [16], nous avons démontré l'inégalité de type Strichartz contenue dans la proposition suivante. Pour montrer que l'on peut choisir des normes différentes sur les diverses parties du terme de force, nous avons écrit $G$ sous la forme $G=G_{1}+G_{2}+G_{3}$.

Proposition 1.3. (Inégalités de Strichartz) Pour tous nombres réels strictement positifs $\delta$, $\delta_{1}, \delta_{2}$ et $\delta_{3}$, il existe une constante strictement positive $C_{4}=C_{4}\left(\delta, \delta_{1}, \delta_{2}, \delta_{3}\right)$ telle que, pour toute solution $U$ du système (1.21) et pour tout $t>0$, on a

$$
\begin{aligned}
\left\|\left(I-\tilde{S}_{0}\right) U\right\|_{L^{2}\left((0, t), L^{\infty}\right)} \leq C_{4}\left(\left\|\left(I-\tilde{S}_{0}\right) u_{0}\right\|_{1+\delta}\right. & +\left\|\left(I-\tilde{S}_{0}\right) u_{1}\right\|_{\delta}+\left\|\left(I-\tilde{S}_{0}\right) G_{1}\right\|_{L^{1}\left((0, t), \dot{H}^{\delta_{1}}\right)} \\
& \left.+\left\|\left(I-\tilde{S}_{0}\right) G_{2}\right\|_{L^{2}\left((0, t), \dot{H}^{\frac{1}{2}+\delta_{2}}\right)}+\left\|\left(I-\tilde{S}_{0}\right) G_{3}\right\|_{L^{p}\left((0, t), \dot{H}^{\frac{p-1}{p}+\delta_{3}}\right)}\right)
\end{aligned}
$$

où $1 \leq p \leq 2$.

Remarquons qu'en dimension trois d'espace, l'estimation de Strichartz classique, pour l'équation des ondes sans dissipation, ne donne pas une estimation de $\left(I-\tilde{S}_{0}\right) U$ en norme $L^{2}\left((0, t), L^{\infty}\right)$ avec une constante $C_{4}$ indépendante de $t$, mais seulement en norme $L^{p}\left((0, t), L^{\infty}\right)$, pour $p>2$. Ici, nous obtenons une estimation de $\left(I-\tilde{S}_{0}\right) U$ en norme $L^{2}\left((0, t), L^{\infty}\right)$ avec une constante $C_{4}$ indépendante de $t$, car nous avons tiré partie de la présence du terme de dissipation $U_{t}$ dans l'équation (1.21) (pour plus de détails, voir [16]). 
Au paragraphe 2, dans la démonstration du théorème 0.4 , nous appliquerons la proposition 1.3 à la solution du système $(1.20)$ avec $G=-\mathbb{P}(u \nabla u)+\mathbb{P} f$. Remarquons que nous aurions aussi pu appliquer l'estimation plus faible dans $L^{p}\left((0, t), L^{\infty}\right)$, pour $p>2$, mais la démonstration aurait été plus longue et plus technique.

\section{DÉmonstration De L'existence Globale de SOlutions Pour Des Données PETITES EN DIMENSION TROIS D'ESPACE}

Soit $0<\delta \leq \frac{1}{2}$ et $d>0$ fixés. Soit $f$ un terme de force appartenant à l'espace $L^{1}\left((0,+\infty), L^{2}\left(\mathbb{R}^{3}\right)^{3}\right) \cap$ $\left.L^{2}\left((0,+\infty), H^{\delta}\left(\mathbb{R}^{3}\right)^{3}\right) \cap L^{p}\left((0,+\infty), \dot{H}^{\frac{p-1}{p}+d}\left(\mathbb{R}^{3}\right)^{3}\right)\right)$, où $1 \leq p \leq 2$ et où $\frac{3}{4}-\frac{1}{p}+\frac{p-1}{2 p}+\frac{d}{2}>0$.

Pour tout couple de vecteurs à divergence nulle $\left(u_{0}, u_{1}\right) \in H^{1+\delta}\left(\mathbb{R}^{3}\right)^{3} \times H^{\delta}\left(\mathbb{R}^{3}\right)^{3}$, il existe une unique solution intégrale $\left(u(t), u_{t}(t)\right) \in C^{0}\left(\left[0, \tau_{0}\right), H^{1+\delta}\left(\mathbb{R}^{3}\right)^{3} \times H^{\delta}\left(\mathbb{R}^{3}\right)^{3}\right)$ des équations $(1.20)$, où $\tau_{0}>0$ dépend de la norme de $\left(u_{0}, u_{1}\right)$ dans $H^{1+\delta}\left(\mathbb{R}^{3}\right) \times H^{\delta}\left(\mathbb{R}^{3}\right)$ et de celle de $\mathbb{P} f$. Si en outre, $f$ est plus régulière et que $\left(u_{0}, u_{1}\right) \in H^{2}\left(\mathbb{R}^{3}\right)^{3} \times H^{1}\left(\mathbb{R}^{3}\right)^{3}$, alors la solution $\left(u(t), u_{t}(t)\right)$ appartient à $C^{0}\left(\left[0, \tau_{0}\right),\left(H^{2}\left(\mathbb{R}^{3}\right) \times H^{1}\left(\mathbb{R}^{3}\right)\right)^{3}\right)$ et est solution classique de (1.20). Soit $T_{0}>0$ le temps maximal d'existence de la solution $\left(u(t), u_{t}(t)\right)$ de donnée initiale $\left(u_{0}, u_{1}\right)$. Si $T_{0}$ est un temps fini, alors la norme $\left\|\left(u(t), u_{t}(t)\right)\right\|_{H^{1+\delta} \times H^{\delta}}$ tend vers l'infini quand $t$ tend vers $T_{0}$. La démonstration de l'existence locale de solutions de (1.20) se fait comme dans [16] en utilisant la forme intégrale de l'équation et le théorème de contraction stricte. On démontre l'unicité de la solution en appliquant le même type d'inégalités que celles utilisées ci-dessous. Comme les démonstrations de l'existence locale et de l'unicité sont analogues à celles données dans [16] dans le cas de la dimension deux d'espace, nous ne les reproduisons pas ici et renvoyons le lecteur à [16] .

Soit donc $\left(u_{0}, u_{1}\right) \in H^{1+\delta}\left(\mathbb{R}^{3}\right) \times H^{\delta}\left(\mathbb{R}^{3}\right)$ un vecteur à divergence nulle. On note $\left(u, u_{t}\right)$ la solution locale de (1.20) de donnée initiale $\left(u_{0}, u_{1}\right)$; on pose

$$
v=\tilde{S}_{0} u, \quad w=\left(I-\tilde{S}_{0}\right) u
$$

On rappelle que, comme conséquence du théorème de Bernstein, $v(t)$ est aussi régulière que voulu.

Soit $k_{0}>0$ une constante donnée petite, qui sera précisée ultérieurement. Si $\|v(0)\|_{L^{\infty}},\|\nabla v(0)\|_{L^{2}},\left\|\nabla v_{t}(0)\right\| L_{L^{2}}$, $\|\Delta v(0)\|_{L^{2}}$ et $\left\|\left(w(0), w_{t}(0)\right)\right\|_{H^{1+\delta} \times H^{\delta}}$ sont suffisamment petits, alors, par continuité, pour $t^{*}>0$ assez petit, $\|v(t)\|_{L^{\infty}},\|\nabla v(t)\|_{L^{2}},\left\|\nabla v_{t}(t)\right\|_{L^{2}},\|\Delta v(t)\|_{L^{2}}$ et $\left\|\left(w(t), w_{t}(t)\right)\right\|_{H^{1+\delta} \times H^{\delta}}$ sont inférieurs à $k_{0} / 10$, par exemple, pour tout $t \in\left[0, t^{*}\right]$. En outre, $\|w(t)\|_{L^{2}\left(\left(0, t^{*}\right), L^{\infty}\right)}$ est aussi inférieur à $k_{0} / 10$. En effet, l'existence locale et les estimations de Strichartz données à la proposition 1.3 entraînent la continuité en temps de la norme de $w$ dans $L^{2}\left((0, t), L^{\infty}\right)$. Il existe donc un temps $0<T^{*} \leq T_{0}$ tel que

$$
T^{*}=\sup \left\{t>0 \mid\|v(t)\|_{L^{\infty}}+\|\Delta v\|_{L^{2}\left((0, t), L^{2}\right)}^{1 / 2}\|\nabla v\|_{L^{2}\left((0, t), L^{2}\right)}^{1 / 2}+\|w\|_{L^{2}\left((0, t), L^{\infty}\right)} \leq k_{0}\right\} .
$$

Supposons que $T_{0}>0$ soit un temps fini et que $T^{*}<T_{0}$. Dans un premier temps, on va établir des estimations a priori de $\left(v(t), v_{t}(t)\right)$ et $\left(w(t), w_{t}(t)\right)$ sur l'intervalle $\left[0, T^{*}\right]$. Ensuite, on montrera par contradiction que $T^{*}>T_{0}$ et que en fait $T^{*}$ et $T_{0}$ sont infinis. Dans une première étape, on va rappeler la fonctionnelle d'énergie classique communément utilisée dans l'étude des équations d'ondes avec dissipation et d'ailleurs aussi utilisée par [3]. A l'aide de variantes de cette fonctionnelle classique, on va établir diverses inégalités d'énergie pour $v$ et $w$. Dans une deuxième étape, on fera la somme de toutes ces inégalités pour obtenir des estimations a priori de $v$ et $w$ sur l'intervalle $\left[0, T^{*}\right]$. Dans la troisième étape, on donnera une estimation a priori de $\|w\|_{L^{2}\left(\left(0, T^{*}\right), L^{\infty}\right)}$. Dans la dernière étape, on montrera qu'en fait $T_{0}=T^{*}=+\infty$.

\subsection{Première étape}

Si on applique l'opérateur $\tilde{S}_{0}$ à l'équation (1.20), on obtient l'équation suivante satisfaite par $v$,

$$
v_{t t}+v_{t}-\nu \Delta v=\tilde{S}_{0} \mathbb{P} f-\tilde{S}_{0} \mathbb{P}(u \cdot \nabla u) .
$$


Si on forme le produit scalaire dans $L^{2}\left(\mathbb{R}^{3}\right)$ de $(2.2)$ avec $v+2 v_{t}$ et si on intègre par parties, on obtient l'égalité suivante, pour $0 \leq t \leq T_{0}$,

$$
\begin{aligned}
\frac{d}{d t}\left(\frac{1}{2}\left\|v+v_{t}\right\|_{L^{2}}^{2}+\frac{1}{2}\left\|v_{t}\right\|_{L^{2}}^{2}+\nu\|\nabla v\|_{L^{2}}^{2}\right)+\left(\left\|v_{t}\right\|_{L^{2}}^{2}+\nu\|\nabla v\|_{L^{2}}^{2}\right) \\
=\left(\tilde{S}_{0}(\mathbb{P} f), v+2 v_{t}\right)-\left(\tilde{S}_{0}(\mathbb{P}(u \cdot \nabla u)), v+2 v_{t}\right) .
\end{aligned}
$$

L'inégalité de Young $|a b| \leq a^{2} / 2 d+b^{2} d / 2$ et l'équation ci-dessus impliquent l'inégalité suivante

$$
\begin{aligned}
& \frac{d}{d t}\left(\frac{1}{2}\left\|v+v_{t}\right\|_{L^{2}}^{2}+\frac{1}{2}\left\|v_{t}\right\|_{L^{2}}^{2}+\nu\|\nabla v\|_{L^{2}}^{2}\right)+\left(\frac{3}{4}\left\|v_{t}\right\|_{L^{2}}^{2}+\nu\|\nabla v\|_{L^{2}}^{2}\right) \\
& \leq\left|\left(\tilde{S}_{0}(\mathbb{P} f), v+v_{t}\right)\right|+\left|\left(\tilde{S}_{0}(\mathbb{P}(u \cdot \nabla u)), v+2 v_{t}\right)\right|+\left\|\tilde{S}_{0} \mathbb{P} f\right\|_{L^{2}}^{2}
\end{aligned}
$$

Si on intègre l'inégalité (2.3) entre 0 et $t$, pour tout $0<t \leq T_{0}$, on obtient l'estimation,

$$
\begin{aligned}
& \frac{1}{2}\left\|\left(v+v_{t}\right)(t)\right\|_{L^{2}}^{2}+\frac{1}{2}\left\|v_{t}(t)\right\|_{L^{2}}^{2}+\nu\|\nabla v(t)\|_{L^{2}}^{2}+\int_{0}^{t}\left(\frac{3}{4}\left\|v_{t}(s)\right\|_{L^{2}}^{2}+\nu\|\nabla v(s)\|_{L^{2}}^{2}\right) d s \\
& \quad \leq \frac{1}{2}\left\|v(0)+v_{t}(0)\right\|_{L^{2}}^{2}+\frac{1}{2}\left\|v_{t}(0)\right\|_{L^{2}}^{2}+\nu\|\nabla v(0)\|_{L^{2}}^{2}+\left\|\tilde{S}_{0} \mathbb{P} f\right\|_{L_{t}^{2}\left(L^{2}\right)}^{2}+\int_{0}^{t}\left|\left(\tilde{S}_{0}(\mathbb{P}(u \cdot \nabla u)), v+2 v_{t}\right)\right| d s \\
& \quad+\left\|\tilde{S}_{0}(\mathbb{P} f)\right\|_{L^{1}\left((0, t), L^{2}\right)}\left\|v+v_{t}\right\|_{L^{\infty}\left((0, t), L^{2}\right)} .
\end{aligned}
$$

De l'inégalité ci-dessus, on déduit en particulier que, pour $0<t \leq T_{0}$,

$$
\begin{aligned}
\frac{1}{4} \|\left(v+v_{t}\right) & \left\|_{L^{\infty}\left((0, t), L^{2}\right)}^{2}+\frac{1}{2}\right\| v_{t}\left\|_{L^{\infty}\left((0, t), L^{2}\right)}^{2}+\nu\right\| \nabla v \|_{L^{\infty}\left((0, t), L^{2}\right)}^{2}+\int_{0}^{t}\left(\frac{3}{4}\left\|v_{t}(s)\right\|_{L^{2}}^{2}+\nu\|\nabla v(s)\|_{L^{2}}^{2}\right) d s \\
\leq & \frac{1}{2}\left\|v(0)+v_{t}(0)\right\|_{L^{2}}^{2}+\frac{1}{2}\left\|v_{t}(0)\right\|_{L^{2}}^{2}+\nu\|\nabla v(0)\|_{L^{2}}^{2}+\left\|\tilde{S}_{0} \mathbb{P} f\right\|_{L^{2}\left(\left(0, T^{*}\right), L^{2}\right)}^{2} \\
& +\int_{0}^{t}\left|\left(\tilde{S}_{0}(\mathbb{P}(u \cdot \nabla u)), v+2 v_{t}\right)\right| d s+\left\|\tilde{S}_{0}(\mathbb{P} f)\right\|_{L^{1}\left(\left(0, T^{*}\right), L^{2}\right)}^{2} \cdot
\end{aligned}
$$

Si on forme maintenant le produit scalaire dans $L^{2}\left(\mathbb{R}^{3}\right)$ de $(2.2)$ avec $-\Delta v-2 \Delta v_{t}$ et si on intègre par parties, on obtient l'égalité suivante, pour $0 \leq t \leq T_{0}$,

$$
\begin{aligned}
\frac{d}{d t}\left(\frac{1}{2}\left\|\nabla\left(v+v_{t}\right)\right\|_{L^{2}}^{2}+\frac{1}{2}\left\|\nabla v_{t}\right\|_{L^{2}}^{2}+\nu\|\Delta v\|_{L^{2}}^{2}\right)+ & \left(\left\|\nabla v_{t}\right\|_{L^{2}}^{2}+\nu\|\Delta v\|_{L^{2}}^{2}\right) \\
& =\left(\tilde{S}_{0}(\mathbb{P} f),-\Delta\left(v+2 v_{t}\right)\right)+\left(\tilde{S}_{0}(\mathbb{P}(u \cdot \nabla u)), \Delta\left(v+2 v_{t}\right)\right)
\end{aligned}
$$

L'inégalité de Young et le lemme de Bernstein ainsi que l'égalité précédente impliquent que, pour $0 \leq t \leq T_{0}$,

$$
\begin{aligned}
\frac{d}{d t}\left(\frac{1}{2}\left\|\nabla\left(v+v_{t}\right)\right\|_{L^{2}}^{2}+\frac{1}{2}\left\|\nabla v_{t}\right\|_{L^{2}}^{2}+\nu\|\Delta v\|_{L^{2}}^{2}\right)+\frac{3}{4}\left(\left\|\nabla v_{t}\right\|_{L^{2}}^{2}+\nu\|\Delta v\|_{L^{2}}^{2}\right) \\
\leq\left(\frac{1}{\nu}\left\|\tilde{S}_{0} \mathbb{P} f\right\|_{L^{2}}^{2}+4\left\|\nabla \tilde{S}_{0} \mathbb{P} f\right\|_{L^{2}}^{2}\right)+\left|\left(\mathbb{P}(u \cdot \nabla u), \Delta\left(v+2 v_{t}\right)\right)\right| \\
\leq\left(\frac{1}{\nu}+2^{2 j_{0}+2} C_{0}^{2}\right)\left\|\tilde{S}_{0} \mathbb{P} f\right\|_{L^{2}}^{2}+\left|\left(\tilde{S}_{0}(\mathbb{P}(u \cdot \nabla u)), \Delta\left(v+2 v_{t}\right)\right)\right| .
\end{aligned}
$$


Si on intègre l'inégalité (2.6) entre 0 et $t$, pour $0<t \leq T_{0}$, on obtient,

$$
\begin{aligned}
\frac{1}{2}\left\|\nabla\left(v+v_{t}\right)(t)\right\|_{L^{2}}^{2}+ & \frac{1}{2}\left\|\nabla v_{t}(t)\right\|_{L^{2}}^{2}+\nu\|\Delta v(t)\|_{L^{2}}^{2}+\frac{3}{4} \int_{0}^{t}\left(\left\|\nabla v_{t}\right\|_{L^{2}}^{2}+\nu\|\Delta v\|_{L^{2}}^{2}\right) d s \\
\leq & \frac{1}{2}\left\|\nabla\left(v+v_{t}\right)(0)\right\|_{L^{2}}^{2}+\frac{1}{2}\left\|\nabla v_{t}(0)\right\|_{L^{2}}^{2}+\nu\|\Delta v(0)\|_{L^{2}}^{2} \\
& +\left(\frac{1}{\nu}+2^{2 j_{0}+2} C_{0}^{2}\right)\left\|\tilde{S}_{0} \mathbb{P} f\right\|_{L^{2}\left((0, t), L^{2}\right)}^{2}+\int_{0}^{t}\left|\left(\tilde{S}_{0}(\mathbb{P}(u \cdot \nabla u)), \Delta\left(v+2 v_{t}\right)\right)\right| d s .
\end{aligned}
$$

Maintenant, nous allons estimer le vecteur $\left(w, w_{t}\right)$ dans $\dot{H}^{s+1}\left(\mathbb{R}^{3}\right) \times \dot{H}^{s}\left(\mathbb{R}^{3}\right)$. Si on applique l'opérateur $I-\tilde{S}_{0}$ à l'équation (1.20), on obtient l'équation suivante en $w$,

$$
w_{t t}+w_{t}-\nu \Delta w=\left(I-\tilde{S}_{0}\right) \mathbb{P} f-\left(I-\tilde{S}_{0}\right) \mathbb{P}(u \cdot \nabla u) .
$$

Si on prend le produit dans $\dot{H}^{\delta}\left(\mathbb{R}^{3}\right)$ de l'équation (2.8) avec $w+2 w_{t}$, on a, pour $0<t \leq T_{0}$,

$$
\begin{aligned}
\frac{d}{d t}\left(\frac{1}{2}\left\|w+w_{t}\right\|_{\delta}^{2}+\frac{1}{2}\left\|w_{t}\right\|_{\delta}^{2}+\nu\|w\|_{\delta+1}^{2}\right)+ & \left(\left\|w_{t}\right\|_{\delta}^{2}+\nu\|w\|_{\delta+1}^{2}\right) \\
& =\left(\left(I-\tilde{S}_{0}\right) \mathbb{P} f, w+2 w_{t}\right)_{\delta}-\left(\left(I-\tilde{S}_{0}\right) \mathbb{P}(u \cdot \nabla u), w+2 w_{t}\right)_{\delta}
\end{aligned}
$$

L'inégalité de Young et les inégalités (1.13) entraînent l'estimation suivante:

$$
\begin{aligned}
\left|\left(\left(I-\tilde{S}_{0}\right) \mathbb{P} f, w+2 w_{t}\right)_{\delta}\right| & \leq\left\|\left(I-\tilde{S}_{0}\right) \mathbb{P} f\right\|_{\delta}\left(\|w\|_{\delta}+2\left\|w_{t}\right\|_{\delta}\right) \\
& \left.\leq\left\|\left(I-\tilde{S}_{0}\right) \mathbb{P} f\right\|_{\delta}\left(C_{1}^{\frac{1+\delta}{2}}\left(\sum_{j \geq j_{0}} 2^{2 j \delta}\left\|\Delta_{j} w\right\|_{L^{2}}^{2}\right)^{\frac{1}{2}}+2\left\|w_{t}\right\|_{\delta}\right)\right) \\
& \leq\left\|\left(I-\tilde{S}_{0}\right) \mathbb{P} f\right\|_{\delta}\left(\frac{C_{1}^{\frac{1+\delta}{2}}}{2^{j_{0}}}\left(\sum_{j \geq j_{0}} 2^{2 j(\delta+1)}\left\|\Delta_{j} w\right\|_{L^{2}}^{2}\right)^{\frac{1}{2}}+2\left\|w_{t}\right\|_{\delta}\right) \\
& \leq\left\|\left(I-\tilde{S}_{0}\right) \mathbb{P} f\right\|_{\delta}\left(\frac{C_{1}^{\frac{3+2 \delta}{2}}}{2^{j_{0}}}\|w\|_{1+\delta}+2\left\|w_{t}\right\|_{\delta}\right) \\
& \leq\left(\frac{C_{1}^{3+2 \delta}}{\nu 2^{2 j_{0}}}+4\right)\left\|\left(I-\tilde{S}_{0}\right) \mathbb{P} f\right\|_{\delta}^{2}+\frac{1}{4}\left\|w_{t}\right\|_{\delta}^{2}+\frac{\nu}{4}\|w\|_{\delta+1}^{2} .
\end{aligned}
$$

L'égalité (2.9) et l'inégalité (2.10) impliquent, que, pour $0 \leq t \leq T_{0}$,

$$
\begin{aligned}
\frac{d}{d t}\left(\frac{1}{2}\left\|w+w_{t}\right\|_{\delta}^{2}+\frac{1}{2}\left\|w_{t}\right\|_{\delta}^{2}+\nu \|\right. & \left.w \|_{\delta+1}^{2}\right)+\frac{3}{4}\left(\left\|w_{t}\right\|_{\delta}^{2}+\nu\|w\|_{\delta+1}^{2}\right) \\
& \leq\left(\frac{C_{1}^{3+2 \delta}}{\nu 2^{2 j_{0}}}+4\right)\left\|\left(I-\tilde{S}_{0}\right) \mathbb{P} f\right\|_{\delta}^{2}+\left|\left(\left(I-\tilde{S}_{0}\right) \mathbb{P}(u \cdot \nabla u), w+2 w_{t}\right)_{\delta}\right| .
\end{aligned}
$$

et aussi, après intégration entre 0 et $t$, pour $0<t \leq T_{0}$,

$$
\begin{aligned}
\frac{1}{2}\left\|\left(w+w_{t}\right)(t)\right\|_{\delta}^{2}+\frac{1}{2}\left\|w_{t}(t)\right\|_{\delta}^{2} & +\nu\|w(t)\|_{\delta+1}^{2}+\frac{3}{4} \int_{0}^{t}\left(\left\|w_{t}\right\|_{\delta}^{2}+\nu\|w\|_{\delta+1}^{2}\right) d s \\
\leq & \frac{1}{2}\left\|\left(w+w_{t}\right)(0)\right\|_{\delta}^{2}+\frac{1}{2}\left\|w_{t}(0)\right\|_{\delta}^{2}+\nu\|w(0)\|_{\delta+1}^{2} \\
& +\left(\frac{C_{1}^{3+2 \delta}}{\nu 2^{2 j_{0}}}+4\right) \int_{0}^{t}\left\|\left(I-\tilde{S}_{0}\right) \mathbb{P} f\right\|_{\delta}^{2} d s+\int_{0}^{t}\left|\left(\left(I-\tilde{S}_{0}\right) \mathbb{P}(u \cdot \nabla u), w+2 w_{t}\right)_{\delta}\right| d s
\end{aligned}
$$




\subsection{Deuxième étape}

Dans cette étape, on détermine des estimations a priori pour $\left(v, v_{t}\right)$ et $\left(w, w_{t}\right)$ en utilisant les inégalités obtenues dans la première étape.

Si on additionne les inégalités (2.7) et (2.12) et si on applique le lemme de Bernstein, on obtient l'estimation suivante, pour tout $t, 0<t \leq T_{0}$,

$$
\begin{aligned}
\frac{1}{2}\left\|\nabla\left(v+v_{t}\right)(t)\right\|_{L^{2}}^{2}+ & \frac{1}{2}\left\|\nabla v_{t}(t)\right\|_{L^{2}}^{2}+\nu\|\Delta v(t)\|_{L^{2}}^{2}+\frac{3}{4} \int_{0}^{t}\left(\left\|\nabla v_{t}\right\|_{L^{2}}^{2}+\nu\|\Delta v\|_{L^{2}}^{2}\right) d s \\
& +\frac{1}{2}\left\|\left(w+w_{t}\right)(t)\right\|_{\delta}^{2}+\frac{1}{2}\left\|w_{t}(t)\right\|_{\delta}^{2}+\nu\|w(t)\|_{\delta+1}^{2}+\frac{3}{4} \int_{0}^{t}\left(\left\|w_{t}\right\|_{\delta}^{2}+\nu\|w\|_{\delta+1}^{2}\right) d s \\
\leq & E_{0}^{*}+\left|\int_{0}^{t}\left(\tilde{S}_{0}(\mathbb{P}(u \cdot \nabla u)), \Delta\left(v+2 v_{t}\right)\right) d s\right|+\left|\int_{0}^{t}\left(\left(I-\tilde{S}_{0}\right) \mathbb{P}(u \cdot \nabla u), w+2 w_{t}\right)_{\delta} d s\right| \\
\leq & E_{0}+\left|\int_{0}^{t}\left(\tilde{S}_{0}(\mathbb{P}(u \cdot \nabla u)), \Delta\left(v+2 v_{t}\right)\right) d s\right|+\left|\int_{0}^{t}\left(\left(I-\tilde{S}_{0}\right) \mathbb{P}(u \cdot \nabla u), w+2 w_{t}\right)_{\delta} d s\right|
\end{aligned}
$$

où

$$
\begin{aligned}
E_{0}^{*}= & \frac{1}{2}\left\|\nabla\left(v+v_{t}\right)(0)\right\|_{L^{2}}^{2}+\frac{1}{2}\left\|\nabla v_{t}(0)\right\|_{L^{2}}^{2}+\nu\|\Delta v(0)\|_{L^{2}}^{2}+\left(\frac{1}{\nu}+2^{2 j_{0}+2} C_{0}^{2}\right)\left\|\tilde{S}_{0} \mathbb{P} f\right\|_{L^{2}\left((0,+\infty), L^{2}\right)}^{2} \\
& +\frac{1}{2}\left\|\left(w+w_{t}\right)(0)\right\|_{\delta}^{2}+\frac{1}{2}\left\|w_{t}(0)\right\|_{\delta}^{2}+\nu\|w(0)\|_{\delta+1}^{2}+\left(\frac{C_{1}^{3+2 \delta}}{\nu 2^{2 j_{0}}}+4\right)\left\|\left(I-\tilde{S}_{0}\right) \mathbb{P} f\right\|_{L^{2}\left((0,+\infty), \dot{H}^{\delta}\right)}^{2}
\end{aligned}
$$

et

$$
\begin{aligned}
E_{0}= & C_{2}^{2}\left[\|\nabla u(0)\|_{L^{2}}^{2}+2^{2 j_{0}} C_{0}^{2}\left\|u_{t}(0)\right\|_{L^{2}}^{2}+2^{2 j_{0}} C_{0}^{2} \nu\|\nabla u(0)\|_{L^{2}}^{2}+\left(\frac{1}{\nu}+2^{2 j_{0}+2} C_{0}^{2}\right)\|\mathbb{P} f\|_{L^{2}\left((0,+\infty), L^{2}\right)}^{2}\right. \\
& \left.+\frac{3}{2}\left\|u_{t}(0)\right\|_{\delta}^{2}+\left(\nu+\frac{C_{1}^{1+2 \delta}}{\nu 2^{2 j_{0}}}\right)\|u(0)\|_{\delta+1}^{2}+\left(\frac{C_{1}^{3+2 \delta}}{\nu 2^{2 j_{0}}}+4\right)\|\mathbb{P} f\|_{L^{2}\left((0,+\infty), \dot{H}^{\delta}\right)}^{2}\right]
\end{aligned}
$$

Nous allons maintenant estimer les deux derniers termes du membre de droite de l'inégalité (2.13). Le terme $\left|\int_{0}^{t}\left(\tilde{S}_{0}(\mathbb{P}(u \cdot \nabla u)), \Delta v+2 \Delta v_{t}\right) d s\right|$ se majore comme suit. On décompose d'abord $u$ en la somme $v+w$ et on écrit,

$$
\left|\int_{0}^{t}\left(\tilde{S}_{0}(\mathbb{P}(u \cdot \nabla u)), \Delta v+2 \Delta v_{t}\right) d s\right|=\left|\int_{0}^{t}\left(\tilde{S}_{0} \mathbb{P}(w \cdot \nabla(v+w)+v \cdot \nabla w+v \cdot \nabla v), \Delta v+2 \Delta v_{t}\right) d s\right|
$$

Ensuite, on majore séparément chaque terme. On obtient alors, pour $0<t \leq T_{0}$,

$$
\begin{aligned}
\int_{0}^{t}\left|\left(\tilde{S}_{0} \mathbb{P}(w \cdot \nabla(v+w)+v \cdot \nabla w), \Delta v+2 \Delta v_{t}\right)\right| d s & \leq C_{2} \int_{0}^{T^{*}}\|w\|_{L^{\infty}}\left(\|\nabla w\|_{L^{2}}+\|\nabla v\|_{L^{2}}\right)\left(\|\Delta v\|_{L^{2}}+2\left\|\Delta v_{t}\right\|_{L^{2}}\right) d s \\
& +C_{2}\|v\|_{L_{t}^{\infty}\left(L^{\infty}\right)} \int_{0}^{T^{*}}\|\nabla w\|_{L^{2}}\left(\|\Delta v\|_{L^{2}}+2\left\|\Delta v_{t}\right\|_{L^{2}}\right) d s \\
\leq C_{2}\|w\|_{L_{t}^{2}\left(L^{\infty}\right)}\left(\|\nabla w\|_{L_{t}^{\infty}\left(L^{2}\right)}\right. & \left.+\|\nabla v\|_{L_{t}^{\infty}\left(L^{2}\right)}\right)\left(\|\Delta v\|_{L_{t}^{2}\left(L^{2}\right)}+2\left\|\Delta v_{t}\right\|_{L_{t}^{2}\left(L^{2}\right)}\right) \\
& +C_{2}\|v\|_{L_{t}^{\infty}\left(L^{\infty}\right)}\|\nabla w\|_{L_{t}^{2}\left(L^{2}\right)}\left(\|\Delta v\|_{L_{t}^{2}\left(L^{2}\right)}+2\left\|\Delta v_{t}\right\|_{L_{t}^{2}\left(L^{2}\right)}\right)
\end{aligned}
$$

où, ici et dans la suite, $L_{t}^{p}\left(L^{q}\right)$ désigne $L^{p}\left((0, t), L^{q}\left(\mathbb{R}^{3}\right)^{3}\right)$. En tenant compte de la définition $(2.1)$ de $T^{*}$, des inégalités de Bernstein, des inégalités (1.13) et de l'inégalité de Young, on déduit de l'inégalité ci-dessus que, 
pour $0<t \leq T^{*}$,

$$
\begin{aligned}
\left|\int_{0}^{t}\left(\tilde{S}_{0} \mathbb{P}(w \cdot \nabla(v+w)+v \cdot \nabla w), \Delta v+2 \Delta v_{t}\right) d s\right| \\
\leq C_{2} k_{0}\left(C_{1}^{2+\delta / 2}\|w\|_{L_{t}^{2}\left(\dot{H}^{\delta+1}\right)}+C_{1}^{2+\delta / 2}\|w\|_{L_{t}^{\infty}\left(\dot{H}^{\delta+1}\right)}+\|\nabla v\|_{L_{t}^{\infty}\left(L^{2}\right)}\right)\left(\|\Delta v\|_{L_{t}^{2}\left(L^{2}\right)}+2^{j_{0}+1} C_{0}\left\|\nabla v_{t}\right\|_{L_{t}^{2}\left(L^{2}\right)}\right) \\
\leq 3 C_{2}^{2} k_{0}^{2}\left(\frac{1}{4 \eta \nu}+\frac{2^{2 j_{0}} C_{0}^{2}}{\eta}\right)\left(C_{1}^{4+\delta}\|w\|_{L_{t}^{2}\left(\dot{H}^{\delta+1}\right)}^{2}+C_{1}^{4+\delta}\|w\|_{L_{t}^{\infty}\left(\dot{H}^{\delta+1}\right)}^{2}+\|\nabla v\|_{L_{t}^{\infty}\left(L^{2}\right)}^{2}\right) \\
\quad+\eta \nu\|\Delta v\|_{L_{t}^{2}\left(L^{2}\right)}^{2}+\eta\left\|\nabla v_{t}\right\|_{L_{t}^{2}\left(L^{2}\right)}^{2} .
\end{aligned}
$$

Il reste à majorer le terme $\left.\mid \int_{0}^{t}\left(\tilde{S}_{0} \mathbb{P} v \cdot \nabla v \cdot \nabla v\right), \Delta v+2 \Delta v_{t}\right) d s \mid$. Grâce à l'inégalité de Hölder et aux inégalités de Sobolev, on peut écrire, pour $0<t \leq T^{*}$,

$$
\begin{aligned}
\left|\int_{0}^{t}\left(\tilde{S}_{0} \mathbb{P}(v \cdot \nabla v), \Delta v+2 \Delta v_{t}\right) d s\right| & \leq C_{2} \int_{0}^{t}\|v\|_{L^{6}}\|\nabla v\|_{L^{3}}\left(\|\Delta v\|_{L^{2}}+2\left\|\Delta v_{t}\right\|_{L^{2}}\right) d s \\
& \leq C_{2} \int_{0}^{t}\|v\|_{L^{6}}\|\nabla v\|_{L^{2}}^{1 / 2}\|\nabla v\|_{L^{6}}^{1 / 2}\left(\|\Delta v\|_{L^{2}}+2\left\|\Delta v_{t}\right\|_{L^{2}}\right) d s \\
& \leq C_{2} C_{S}^{3 / 2} \int_{0}^{t}\|\nabla v\|_{L^{2}}^{3 / 2}\|\Delta v\|_{L^{2}}^{1 / 2}\left(\|\Delta v\|_{L^{2}}+2\left\|\Delta v_{t}\right\|_{L^{2}}\right) d s \\
& \leq C_{2} C_{S}^{3 / 2}\|\nabla v\|_{L_{t}^{\infty}\left(L^{2}\right)}\|\nabla v\|_{L_{t}^{2}\left(L^{2}\right)}^{1 / 2}\|\Delta v\|_{L_{t}^{2}\left(L^{2}\right)}^{1 / 2}\left(\|\Delta v\|_{L_{t}^{2}\left(L^{2}\right)}+2\left\|\Delta v_{t}\right\|_{L_{t}^{2}\left(L^{2}\right)}\right)
\end{aligned}
$$

En tenant compte de la définition (2.1) de $T^{*}$ et en appliquant les inégalités de Bernstein et l'inégalité de Young, on déduit de l'inégalité ci-dessus que, pour $0<t \leq T^{*}$,

$$
\begin{aligned}
\mid \int_{0}^{t}\left(\tilde{S}_{0} \mathbb{P}(v \cdot \nabla v), \Delta v+\right. & \left.2 \Delta v_{t}\right) d s \mid \\
& \leq C_{2} C_{S}^{3 / 2}\|\nabla v\|_{L_{t}^{\infty}\left(L^{2}\right)}\|\nabla v\|_{L_{t}^{2}\left(L^{2}\right)}^{1 / 2}\|\Delta v\|_{L_{t}^{2}\left(L^{2}\right)}^{1 / 2}\left(\|\Delta v\|_{L_{t}^{2}\left(L^{2}\right)}+2^{j_{0}+1} C_{0}\left\|\Delta v_{t}\right\|_{L_{t}^{2}\left(L^{2}\right)}\right) \\
& \leq k_{0} C_{2} C_{S}^{3 / 2}\|\nabla v\|_{L_{t}^{\infty}\left(L^{2}\right)}\left(\|\Delta v\|_{L_{t}^{2}\left(L^{2}\right)}+2^{j_{0}+1} C_{0}\left\|\nabla v_{t}\right\|_{L_{t}^{2}\left(L^{2}\right)}\right) \\
& \leq\left(\frac{k_{0}^{2} C_{2}^{2} C_{S}^{3}}{4 \eta \nu}+\frac{k_{0}^{2} C_{2}^{2} C_{S}^{3} 2^{2 j_{0}} C_{0}^{2}}{\eta}\right)\|\nabla v\|_{L_{t}^{\infty}\left(L^{2}\right)}^{2}+\eta \nu\|\Delta v\|_{L_{t}^{2}\left(L^{2}\right)}^{2}+\eta\left\|\nabla v_{t}\right\|_{L_{t}^{2}\left(L^{2}\right)}^{2} .
\end{aligned}
$$

Pour majorer le terme $\left|\int_{0}^{t}\left(\left(I-\tilde{S}_{0}\right) \mathbb{P}(u \cdot \nabla u), w+2 w_{t}\right)_{\delta} d s\right|$, on décompose à nouveau $u$ en la somme $v+w$. Mais maintenant, on utilise la loi de produit rappelée dans la proposition 1.2 et ainsi on obtient

$$
\begin{aligned}
\left|\int_{0}^{t}\left(\left(I-\tilde{S}_{0}\right) \mathbb{P}(w \cdot \nabla(v+w)+v \cdot \nabla w), w+2 w_{t}\right)_{\delta} d s\right| \\
\quad \leq \int_{0}^{T^{*}}\left(\left\|\left(I-\tilde{S}_{0}\right)(w \cdot \nabla v)\right\|_{\delta}+\left\|\left(I-\tilde{S}_{0}\right)(w \cdot \nabla w)\right\|_{\delta}+\left\|\left(I-\tilde{S}_{0}\right)(v \cdot \nabla w)\right\|_{\delta}\right)\left\|w+2 w_{t}\right\|_{\delta} d s \\
\quad \leq 2 C_{2} \int_{0}^{T^{*}}\left(\|w\|_{L^{\infty}}\|v\|_{\delta+1}+\|v\|_{L^{\infty}}\|w\|_{\delta+1}+\|w\|_{L^{\infty}}\|w\|_{\delta+1}\right)\left\|w+2 w_{t}\right\|_{\delta} d s \\
\quad \leq 2 C_{2}\left(\|w\|_{L_{t}^{2}\left(L^{\infty}\right)}\|v\|_{L_{t}^{\infty}\left(\dot{H}^{\delta+1}\right)}+\|v\|_{L_{t}^{\infty}\left(L^{\infty}\right)}\|w\|_{L_{t}^{2}\left(\dot{H}^{\delta+1}\right)}+\|w\|_{L_{t}^{2}\left(L^{\infty}\right)}\|w\|_{L_{t}^{\infty}\left(\dot{H}^{\delta+1}\right)}\right)\left\|w+2 w_{t}\right\|_{L_{t}^{2}\left(\dot{H}^{\delta}\right)} .
\end{aligned}
$$


Grâce à la définition (2.1) de $T^{*}$, l'inégalité ci-dessus implique que

$$
\begin{aligned}
\mid \int_{0}^{t}\left(\left(I-\tilde{S}_{0}\right) \mathbb{P}(w \cdot \nabla(v+w)\right. & \left.+v \cdot \nabla w), w+2 w_{t}\right)_{\delta} d s \mid \\
& \leq 2 C_{2} k_{0}\left(\|v\|_{L_{t}^{\infty}\left(\dot{H}^{\delta+1}\right)}+\|w\|_{L_{t}^{2}\left(\dot{H}^{\delta+1}\right)}+\|w\|_{L_{t}^{\infty}\left(\dot{H}^{\delta+1}\right)}\right)\left(\|w\|_{L_{t}^{2}\left(\dot{H}^{\delta}\right)}+2\left\|w_{t}\right\|_{L_{t}^{2}\left(\dot{H}^{\delta}\right)}\right)
\end{aligned}
$$

En appliquant plusieurs fois l'inégalité (1.13) ainsi que l'inégalité de Young, on déduit de l'estimation ci-dessus que, pour $0<t \leq T^{*}$,

$$
\begin{aligned}
\mid \int_{0}^{t}\left(\left(I-\tilde{S}_{0}\right)\right. & \left.\mathbb{P}(w \cdot \nabla(v+w)+v \cdot \nabla w), w+2 w_{t}\right)_{\delta} d s \mid \\
\leq & 2 k_{0} C_{2}\left(\|v\|_{L_{t}^{\infty}\left(\dot{H}^{\delta+1}\right)}+\|w\|_{L_{t}^{2}\left(\dot{H}^{\delta+1}\right)}+\|w\|_{L_{t}^{\infty}\left(\dot{H}^{\delta+1}\right)}\right)\left(C_{1}^{\delta+3 / 2}\|w\|_{L_{t}^{2}\left(\dot{H}^{1+\delta}\right)}+2\left\|w_{t}\right\|_{L_{t}^{2}\left(\dot{H}^{\delta}\right)}\right) \\
\leq & C_{2}^{2}\left(\frac{3 k_{0}^{2} C_{1}^{3+2 \delta}}{\eta \nu}+\frac{12 k_{0}^{2}}{\eta}\right)\left(C_{1}^{4+\delta} 2^{2 j_{0} \delta}\|\nabla v\|_{L_{t}^{\infty}\left(L^{2}\right)}^{2}+\|w\|_{L_{t}^{2}\left(\dot{H}^{\delta+1}\right)}^{2}+\|w\|_{L_{t}^{\infty}\left(\dot{H}^{\delta+1}\right)}^{2}\right) \\
& +\eta \nu\|w\|_{L_{t}^{2}\left(\dot{H}^{1+\delta}\right)}^{2}+\eta\left\|w_{t}\right\|_{L_{t}^{2}\left(\dot{H}^{\delta}\right)}^{2} .
\end{aligned}
$$

Il reste à majorer le terme $\left|\int_{0}^{t}\left(\left(I-\tilde{S}_{0}\right) \mathbb{P}(v \cdot \nabla v), w+2 w_{t}\right)_{\delta} d s\right|$. Puisque le terme $\left(I-\tilde{S}_{0}\right) \mathbb{P}(v \cdot \nabla v)$ ne contient que des hautes fréquences, on peut appliquer les inégalités (1.13), c'est-à-dire remplacer le terme $\left\|\left(I-\tilde{S}_{0}\right) \mathbb{P}(v \cdot \nabla v)\right\|_{\delta}$ par $\left\|\left(I-\tilde{S}_{0}\right) \mathbb{P}(v \cdot \nabla v)\right\|_{1}$ par exemple. En appliquant en outre les inégalités (1.13) et la proposition 1.2 , on obtient alors les estimations suivantes, pour $0<t \leq T_{0}$,

$$
\begin{aligned}
\left|\int_{0}^{t}\left(\left(I-\tilde{S}_{0}\right) \mathbb{P}(v \cdot \nabla v), w+2 w_{t}\right)_{\delta} d s\right| & \leq \int_{0}^{t}\left\|\left(I-\tilde{S}_{0}\right) \mathbb{P}(v \cdot \nabla v)\right\|_{\delta}\left\|w+2 w_{t}\right\|_{\delta} d s \\
& \leq C_{2} C_{1}^{(3+\delta) / 2} \int_{0}^{t}\|(v \cdot \nabla v)\|_{1}\left\|w+2 w_{t}\right\|_{\delta} d s \\
& \leq 2 C_{3} C_{2} C_{1}^{(3+\delta) / 2} \int_{0}^{t}\|v\|_{L^{\infty}}\|v\|_{2}\left\|w+2 w_{t}\right\|_{\delta} d s \\
& \leq 2 C_{3} C_{2} C_{5} C_{1}^{(3+\delta) / 2}\|v\|_{L_{t}^{\infty}\left(L^{\infty}\right)}\|\Delta v\|_{L^{2}\left(L^{2}\right)}\left\|w+2 w_{t}\right\|_{L^{2}\left(\dot{H}^{\delta}\right)}
\end{aligned}
$$

où $C_{5}$ est une constante strictement positive ne dépendant que de la fonction de troncature $\varphi$ introduite au paragraphe 1.2. Comme dans (2.18), en utilisant deux fois l'inégalité (1.13) et l'inégalité de Young et en tenant compte de la définition de $T^{*}$, on déduit de l'estimation ci-dessus que, pour $0<t \leq T^{*}$,

$$
\begin{gathered}
\left|\int_{0}^{t}\left(\left(I-\tilde{S}_{0}\right) \mathbb{P}(v \cdot \nabla v), w+2 w_{t}\right)_{\delta} d s\right| \leq 2 C_{3} C_{2} C_{5} k_{0} C_{1}^{(3+\delta) / 2}\|\Delta v\|_{L^{2}\left(L^{2}\right)}\left(C_{1}^{\delta+3 / 2}\|w\|_{L_{t}^{2}\left(\dot{H}^{1+\delta}\right)}+2\left\|w_{t}\right\|_{L_{t}^{2}\left(\dot{H}^{\delta}\right)}\right) \\
\leq \eta \nu\|w\|_{L_{t}^{2}\left(\dot{H}^{1+\delta}\right)}^{2}+\eta\left\|w_{t}\right\|_{L_{t}^{2}\left(\dot{H}^{\delta}\right)}^{2}+\frac{\left(k_{0} C_{2} C_{3} C_{5}\right)^{2} C_{1}^{3+\delta}}{\eta}\left(4+\frac{C_{1}^{3+\delta}}{\nu}\|\Delta v\|_{L_{t}^{2}\left(L^{2}\right)}^{2}\right.
\end{gathered}
$$

On pose maintenant $\eta=1 / 8$. Si on majore $\|\nabla v\|_{L_{t}^{\infty}\left(L^{2}\right)}^{2}$ par $2\left\|\nabla v+\nabla v_{t}\right\|_{L_{t}^{\infty}\left(L^{2}\right)}^{2}+2\left\|\nabla v_{t}\right\|_{L_{t}^{\infty}\left(L^{2}\right)}^{2}$, on voit facilement que les estimations (2.13), (2.16), (2.17), (2.18) et (2.19) entraînent l'inégalité suivante, pour 
$0<t \leq T^{*}$

$$
\begin{aligned}
& \frac{1}{2}\left\|\nabla\left(v+v_{t}\right)(t)\right\|_{L^{2}}^{2}+\frac{1}{2}\left\|\nabla v_{t}(t)\right\|_{L^{2}}^{2}+\nu\|\Delta v(t)\|_{L^{2}}^{2}+\frac{1}{2} \int_{0}^{t}\left(\left\|\nabla v_{t}\right\|_{L^{2}}^{2}+\nu\|\Delta v\|_{L^{2}}^{2}\right) d s \\
&+\frac{1}{2}\left\|\left(w+w_{t}\right)(t)\right\|_{\delta}^{2}+\frac{1}{2}\left\|w_{t}(t)\right\|_{\delta}^{2}+\nu\|w(t)\|_{\delta+1}^{2}+\frac{1}{2} \int_{0}^{t}\left(\left\|w_{t}\right\|_{\delta}^{2}+\nu\|w\|_{\delta+1}^{2}\right) d s \\
& \leq E_{0}+k_{0}^{2} \frac{\left(C_{2} C_{3} C_{5}\right)^{2} C_{1}^{3+\delta}}{\eta}\left(4+\frac{C_{1}^{3+\delta}}{\nu}\right)\|\Delta v\|_{L_{t}^{2}\left(L^{2}\right)}^{2} \\
&+2 k_{0}^{2} C_{2}^{2}\left[3\left(\frac{1}{4 \eta \nu}+\frac{2^{2 j_{0}} C_{0}^{2}}{\eta}\right)+\left(\frac{C_{S}^{3}}{4 \eta \nu}+\frac{C_{S}^{3} 2^{2 j_{0}} C_{0}^{2}}{\eta}\right)+\left(\frac{3 C_{1}^{3+2 \delta}}{\eta \nu}+\frac{12}{\eta}\right) C_{1}^{4+\delta} 2^{2 j_{0} \delta}\right] \\
&+3 k_{0}^{2} C_{2}^{2}\left[C_{1}^{4+\delta}\left(\frac{1}{4 \eta \nu}+\frac{2^{2 j_{0}} C_{0}^{2}}{\eta}\right)+\left(\frac{C_{1}^{3+2 \delta}}{\eta \nu}+\frac{4}{\eta}\right)\right]\left(\|w\|_{L_{t}^{2}\left(\dot{H}^{\delta+1}\right)}^{2}+\|w\|_{L_{t}^{\infty}\left(\dot{H}^{\delta+1}\right)}^{2}\right)
\end{aligned}
$$

où $\eta=1 / 8$.

Si on choisit maintenant $k_{0}>0$ tel que

$$
\begin{aligned}
k_{0}^{2}\left[\frac{\left(C_{2} C_{3} C_{5}\right)^{2} C_{1}^{3+\delta}}{\eta}\left(4+\frac{C_{1}^{3+\delta}}{\nu}\right)\right] & \leq \frac{\nu}{4} \\
2 k_{0}^{2} C_{2}^{2}\left[3\left(\frac{1}{4 \eta \nu}+\frac{2^{2 j_{0}} C_{0}^{2}}{\eta}\right)+\left(\frac{C_{S}^{3}}{4 \eta \nu}+\frac{C_{S}^{3} 2^{2 j_{0}} C_{0}^{2}}{\eta}\right)+\left(\frac{3 C_{1}^{3+2 \delta}}{\eta \nu}+\frac{12}{\eta}\right) C_{1}^{4+\delta} 2^{2 j_{0} \delta}\right] & \leq \frac{1}{4} \\
3 k_{0}^{2} C_{2}^{2}\left[C_{1}^{4+\delta}\left(\frac{1}{4 \eta \nu}+\frac{2^{2 j_{0}}}{\eta}\right)+\left(\frac{C_{1}^{3+2 \delta}}{\eta \nu}+\frac{4}{\eta}\right)\right] & \leq \frac{\nu}{4},
\end{aligned}
$$

on déduit de l'estimation (2.20) que, pour $0<t \leq T^{*}$,

$$
\begin{aligned}
\frac{1}{4}\left\|\nabla\left(v+v_{t}\right)(t)\right\|_{L^{2}}^{2}+\frac{1}{4}\left\|\nabla v_{t}(t)\right\|_{L^{2}}^{2}+\nu\|\Delta v(t)\|_{L^{2}}^{2}+\int_{0}^{t}\left(\frac{1}{2}\left\|\nabla v_{t}\right\|_{L^{2}}^{2}+\frac{\nu}{4}\|\Delta v\|_{L^{2}}^{2}\right) d s & \\
& +\left\|\left(w+w_{t}\right)(t)\right\|_{\delta}^{2}+\left\|w_{t}(t)\right\|_{\delta}^{2}+\frac{3 \nu}{4}\|w(t)\|_{\delta+1}^{2}+\frac{1}{4} \int_{0}^{t}\left(\left\|w_{t}\right\|_{\delta}^{2}+\nu\|w\|_{\delta+1}^{2}\right) d s \\
& \leq E_{0},
\end{aligned}
$$

ou encore finalement,

$$
\begin{aligned}
\left\|\nabla\left(v+v_{t}\right)(t)\right\|_{L^{2}}^{2}+\left\|\nabla v_{t}(t)\right\|_{L^{2}}^{2} & +\nu\|\Delta v(t)\|_{L^{2}}^{2}+\int_{0}^{t}\left(\left\|\nabla v_{t}\right\|_{L^{2}}^{2}+\nu\|\Delta v\|_{L^{2}}^{2}\right) d s \\
& +\left\|\left(w+w_{t}\right)(t)\right\|_{\delta}^{2}+\left\|w_{t}(t)\right\|_{\delta}^{2}+\nu\|w(t)\|_{\delta+1}^{2}+\int_{0}^{t}\left(\left\|w_{t}\right\|_{\delta}^{2}+\nu\|w\|_{\delta+1}^{2}\right) d s \\
& \leq 4 E_{0} .
\end{aligned}
$$


Pour obtenir des bornes de $\|v\|_{L_{t}^{\infty}\left(L^{2}\right)},\left\|v_{t}\right\|_{L_{t}^{\infty}\left(L^{2}\right)}$ et de $\|\nabla v\|_{L_{t}^{\infty}\left(L^{2}\right)}$, on additionne les inégalités (2.5) et (2.22). On obtient alors, pour $0<t \leq T^{*}$,

$$
\begin{aligned}
& \frac{1}{4}\left\|v+v_{t}\right\|_{L^{\infty}\left((0, t), L^{2}\right)}^{2}+\frac{1}{2}\left\|v_{t}\right\|_{L^{\infty}\left((0, t), L^{2}\right)}^{2}+\nu\|\nabla v\|_{L^{\infty}\left((0, t), L^{2}\right)}^{2}+\int_{0}^{t}\left(\frac{3}{4}\left\|v_{t}(s)\right\|_{L^{2}}^{2}+\nu\|\nabla v(s)\|_{L^{2}}^{2}\right) d s \\
& +\frac{1}{4}\left\|\nabla\left(v+v_{t}\right)\right\|_{L^{\infty}\left((0, t), L^{2}\right)}^{2}+\frac{1}{4}\left\|\nabla v_{t}\right\|_{L^{\infty}\left((0, t), L^{2}\right)}^{2}+\nu\|\Delta v\|_{L^{\infty}\left((0, t), L^{2}\right)}^{2}+\int_{0}^{t}\left(\frac{1}{2}\left\|\nabla v_{t}\right\|_{L^{2}}^{2}+\frac{\nu}{4}\|\Delta v\|_{L^{2}}^{2}\right) d s \\
& +\frac{1}{2}\left\|w+w_{t}\right\|_{L^{\infty}\left((0, t), \dot{H}^{\delta}\right)}^{2}+\frac{1}{2}\left\|w_{t}\right\|_{\left.L^{\infty}\left((0, t), \dot{H}^{\delta}\right)\right)}^{2}+\frac{3 \nu}{4}\|w\|_{L^{\infty}\left((0, t), \dot{H}^{\delta+1}\right)}^{2}+\frac{1}{4} \int_{0}^{t}\left(\left\|w_{t}\right\|_{\delta}^{2}+\nu\|w\|_{\delta+1}^{2}\right) d s \\
& \leq E_{0}+E_{1}^{*}+\int_{0}^{t}\left|\left(\tilde{S}_{0}(\mathbb{P}(u \cdot \nabla u)), v+2 v_{t}\right)\right| d s \leq E_{0}+E_{1}+\int_{0}^{t}\left|\left(\tilde{S}_{0}(\mathbb{P}(u \cdot \nabla u)), v+2 v_{t}\right)\right| d s
\end{aligned}
$$

où

$$
E_{1}^{*}=\frac{1}{2}\left\|v(0)+v_{t}(0)\right\|_{L^{2}}^{2}+\frac{1}{2}\left\|v_{t}(0)\right\|_{L^{2}}^{2}+\nu\|\nabla v(0)\|_{L^{2}}^{2}+\left\|\tilde{S}_{0} \mathbb{P} f\right\|_{L^{2}\left((0,+\infty), L^{2}\right)}^{2}+\left\|\tilde{S}_{0}(\mathbb{P} f)\right\|_{L^{1}\left((0,+\infty), L^{2}\right)}^{2}
$$

et

$$
E_{1}=C_{2}^{2}\left[\frac{1}{2}\left\|u(0)+u_{t}(0)\right\|_{L^{2}}^{2}+\frac{1}{2}\left\|u_{t}(0)\right\|_{L^{2}}^{2}+\nu\|\nabla u(0)\|_{L^{2}}^{2}+\|\mathbb{P} f\|_{L^{2}\left((0,+\infty), L^{2}\right)}^{2}+\|\mathbb{P} f\|_{L^{1}\left((0,+\infty), L^{2}\right)}^{2}\right] .
$$

En tenant compte de la définition de $T^{*}$ et en utilisant l'inégalité de Young ainsi que les inégalités (1.13), on obtient la majoration suivante, pour $0<t \leq T^{*}$,

$$
\begin{aligned}
\int_{0}^{t}\left|\left(\tilde{S}_{0} \mathbb{P}(w \cdot \nabla(v+w)), v+2 v_{t}\right)\right| d s & \leq C_{2} \int_{0}^{t}\|w\|_{L^{\infty}}\left(\|\nabla v\|_{L^{2}}+\|\nabla w\|_{L^{2}}\right)\left\|v+2 v_{t}\right\|_{L^{2}} d s \\
& \leq C_{2}\|w\|_{L_{t}^{2}\left(L^{\infty}\right)}\left(\|\nabla v\|_{L_{t}^{2}\left(L^{2}\right)}+\|\nabla w\|_{L_{t}^{2}\left(L^{2}\right)}\right)\left\|v+2 v_{t}\right\|_{L_{t}^{\infty}\left(L^{2}\right)} \\
& \leq k_{0} C_{2}\left(\|\nabla v\|_{L_{t}^{2}\left(L^{2}\right)}+\|\nabla w\|_{L_{t}^{2}\left(L^{2}\right)}\right)\left(\left\|v+v_{t}\right\|_{L_{t}^{\infty}\left(L^{2}\right)}+\left\|v_{t}\right\|_{L_{t}^{\infty}\left(L^{2}\right)}\right) \\
\leq & \frac{1}{16}\left(\left\|v+v_{t}\right\|_{L_{t}^{\infty}\left(L^{2}\right)}^{2}+\left\|v_{t}\right\|_{L_{t}^{\infty}\left(L^{2}\right)}^{2}\right)+8 k_{0}^{2} C_{2}^{2}\left(\|\nabla v\|_{L_{t}^{2}\left(L^{2}\right)}^{2}+C_{1}^{4+\delta}\|w\|_{L_{t}^{2}\left(\dot{H}^{\delta+1}\right)}^{2}\right)
\end{aligned}
$$

Grâce aux inclusions de Sobolev classiques, en tenant compte de la définition de $T^{*}$ et en utilisant l'inégalité de Young, on majore le terme $\int_{0}^{t} \mid\left(\tilde{S}_{0}\left(\mathbb{P}(v \cdot \nabla v), v+2 v_{t}\right) \mid d s\right.$ de la manière suivante, pour $0<t \leq T^{*}$,

$$
\begin{aligned}
\int_{0}^{t}\left|\left(\tilde{S}_{0} \mathbb{P}(v \cdot \nabla v), v+2 v_{t}\right)\right| d s & \leq C_{2} \int_{0}^{t}\|v\|_{L^{6}}\|\nabla v\|_{L^{3}}\left\|v+2 v_{t}\right\|_{L^{2}} d s \\
& \leq C_{2} \int_{0}^{t}\|v\|_{L^{6}}\|\nabla v\|_{L^{2}}^{1 / 2}\|\nabla v\|_{L^{6}}^{1 / 2}\left\|v+2 v_{t}\right\|_{L^{2}} d s \\
& \leq C_{2} C_{S}^{3 / 2} \int_{0}^{t}\|\nabla v\|_{L^{2}}^{3 / 2}\|\Delta v\|_{L^{2}}^{1 / 2}\left\|v+2 v_{t}\right\|_{L^{2}} d s \\
& \leq C_{2} C_{S}^{3 / 2}\|\nabla v\|_{L_{t}^{2}\left(L^{2}\right)}\|\nabla v\|_{L_{t}^{2}\left(L^{2}\right)}^{1 / 2}\|\Delta v\|_{L_{t}^{2}\left(L^{2}\right)}^{1 / 2}\left(\left\|v+v_{t}\right\|_{L_{t}^{\infty}\left(L^{2}\right)}+\left\|v_{t}\right\|_{L_{t}^{\infty}\left(L^{2}\right)}\right) \\
& \leq C_{2} C_{S}^{3 / 2} k_{0}\|\nabla v\|_{L_{t}^{2}\left(L^{2}\right)}\left(\left\|v+v_{t}\right\|_{L_{t}^{\infty}\left(L^{2}\right)}+\left\|v_{t}\right\|_{L_{t}^{\infty}\left(L^{2}\right)}\right) \\
& \leq \frac{1}{16}\left\|v+v_{t}\right\|_{L_{t}^{\infty}\left(L^{2}\right)}^{2}+\frac{1}{16}\left\|v_{t}\right\|_{L_{t}^{\infty}\left(L^{2}\right)}^{2}+4 k_{0}^{2} C_{S}^{3} C_{2}^{2}\|\nabla v\|_{L_{t}^{2}\left(L^{2}\right)}^{2}
\end{aligned}
$$


Il reste à majorer le terme $\int_{0}^{t}\left|\left(\tilde{S}_{0} \mathbb{P}(v \cdot \nabla w), v+2 v_{t}\right)\right| d s$. On remarque d'abord que, par intégration par parties, on a

$$
\left(\tilde{S}_{0} \mathbb{P}(v \cdot \nabla w), v+2 v_{t}\right)=\left((v \cdot \nabla w), \tilde{S}_{0}\left(v+2 v_{t}\right)\right)=-\left(\left(v \cdot \nabla \tilde{S}_{0}\left(v+2 v_{t}\right)\right), w\right) .
$$

Les inégalités (1.13) et la définition de $T^{*}$ impliquent alors que, pour $0<t \leq T^{*}$,

$$
\begin{aligned}
\int_{0}^{t}\left|\left(\tilde{S}_{0} \mathbb{P}(v \cdot \nabla w), v+2 v_{t}\right)\right| d s & \leq C_{2} \int_{0}^{t}\|v\|_{L^{\infty}}\|w\|_{L^{2}}\left\|\nabla\left(v+2 v_{t}\right)\right\|_{L^{2}} d s \\
& \leq C_{2} C_{1}^{\frac{3+\delta}{2}} \int_{0}^{t}\|v\|_{L^{\infty}}\|w\|_{1+\delta}\left(\|\nabla v\|_{L^{2}}+2\left\|\nabla v_{t}\right\|_{L^{2}}\right) d s \\
& \leq C_{2} C_{1}^{\frac{3+\delta}{2}}\|v\|_{L_{t}^{\infty}\left(L^{\infty}\right)}\|w\|_{L_{t}^{2}\left(H^{\delta+1}\right)}\left(\|\nabla v\|_{L_{t}^{2}\left(L^{2}\right)}+2\left\|\nabla v_{t}\right\|_{L_{t}^{2}\left(L^{2}\right)}\right) \\
& \leq \frac{1}{4}\left\|\nabla v_{t}\right\|_{L_{t}^{2}\left(L^{2}\right)}^{2}+\frac{\nu}{4}\|\nabla v\|_{L_{t}^{2}\left(L^{2}\right)}^{2}+C_{2}^{2} C_{1}^{3+\delta} k_{0}^{2}\left(\frac{1}{\nu}+4\right)\|w\|_{L_{t}^{2}\left(\dot{H}^{\delta+1}\right)}^{2} .
\end{aligned}
$$

Les estimations $(2.24),(2.27),(2.28)$ et $(2.29)$ entrainent, pour $0<t \leq T^{*}$,

$$
\begin{gathered}
\frac{1}{8}\left\|v+v_{t}\right\|_{L^{\infty}\left((0, t), L^{2}\right)}^{2}+\frac{3}{8}\left\|v_{t}\right\|_{L^{\infty}\left((0, t), L^{2}\right)}^{2}+\nu\|\nabla v\|_{L^{\infty}\left((0, t), L^{2}\right)}^{2}+\frac{3}{4} \int_{0}^{t}\left(\left\|v_{t}(s)\right\|_{L^{2}}^{2}+\nu\|\nabla v(s)\|_{L^{2}}^{2}\right) d s \\
+\frac{1}{4}\left\|\nabla\left(v+v_{t}\right)\right\|_{L^{\infty}\left((0, t), L^{2}\right)}^{2}+\frac{1}{4}\left\|\nabla v_{t}\right\|_{L^{\infty}\left((0, t), L^{2}\right)}^{2}+\nu\|\Delta v\|_{L^{\infty}\left((0, t), L^{2}\right)}^{2}+\frac{1}{4} \int_{0}^{t}\left(\left\|\nabla v_{t}\right\|_{L^{2}}^{2}+\nu\|\Delta v\|_{L^{2}}^{2}\right) d s \\
+\frac{1}{2}\left\|w+w_{t}\right\|_{L^{\infty}\left((0, t), \dot{H}^{\delta}\right)}^{2}+\frac{1}{2}\left\|w_{t}\right\|_{\left.L^{\infty}\left((0, t), \dot{H}^{\delta}\right)\right)}^{2}+\frac{3 \nu}{4}\|w\|_{L^{\infty}\left((0, t), \dot{H}^{\delta+1}\right)}^{2}+\frac{1}{4} \int_{0}^{t}\left(\left\|w_{t}\right\|_{\delta}^{2}+\nu\|w\|_{\delta+1}^{2}\right) d s \\
\leq E_{0}+E_{1}+4 k_{0}^{2} C_{2}^{2}\left(2+C_{S}^{3}\right)\|\nabla v\|_{L_{t}^{2}\left(L^{2}\right)}^{2}+k_{0}^{2} C_{2}^{2} C_{1}^{3+\delta}\left(8 C_{1}+4+\frac{1}{\nu}\right)\|w\|_{L_{t}^{2}\left(\dot{H}^{\delta+1}\right)}^{2} .
\end{gathered}
$$

Si on choisit $k_{0}>0$ satisfaisant, en outre, aux conditions

$$
4 k_{0}^{2} C_{2}^{2}\left(2+C_{S}^{3}\right) \leq \frac{\nu}{4}, \quad k_{0}^{2} C_{2}^{2} C_{1}^{3+\delta}\left(8 C_{1}+4+\frac{1}{\nu}\right) \leq \frac{\nu}{8},
$$

on déduit de l'estimation (2.30) que, pour $0<t \leq T *$,

$$
\begin{array}{r}
\frac{1}{8}\left\|v+v_{t}\right\|_{L^{\infty}\left((0, t), L^{2}\right)}^{2}+\frac{3}{8}\left\|v_{t}\right\|_{L^{\infty}\left((0, t), L^{2}\right)}^{2}+\nu\|\nabla v\|_{L^{\infty}\left((0, t), L^{2}\right)}^{2}+\frac{1}{2} \int_{0}^{t}\left(\left\|v_{t}(s)\right\|_{L^{2}}^{2}+\nu\|\nabla v(s)\|_{L^{2}}^{2}\right) d s \\
\leq E_{0}+E_{1},
\end{array}
$$

ou encore,

$$
\left\|v+v_{t}\right\|_{L^{\infty}\left((0, t), L^{2}\right)}^{2}+\left\|v_{t}\right\|_{L^{\infty}\left((0, t), L^{2}\right)}^{2}+\nu\|\nabla v\|_{L^{\infty}\left((0, t), L^{2}\right)}^{2}+\left\|v_{t}(s)\right\|_{L_{t}^{2}\left(L^{2}\right)}^{2}+\nu\|\nabla v(s)\|_{L^{2}\left(L^{2}\right)}^{2} \leq 8\left(E_{0}+E_{1}\right) .
$$

D'après l'inégalité d'Agmon, on a la majoration, pour $0<t \leq T^{*}$,

$$
\|v\|_{L^{\infty}\left((0, t), L^{\infty}\right)} \leq C_{A}\|v\|_{L^{\infty}\left((0, t), L^{2}\right)}^{1 / 4}\|\Delta v\|_{L^{\infty}\left((0, t), L^{2}\right)}^{3 / 4} .
$$

Il s'ensuit des estimations (2.23) et (2.33) que, pour $0<t \leq T^{*}$,

$$
\|v\|_{L^{\infty}\left((0, t), L^{\infty}\right)} \leq 4 C_{A}\left(\frac{8}{\nu}\right)^{3 / 8} E_{0}^{3 / 8}\left(E_{0}+E_{1}\right)^{1 / 8} .
$$




\subsection{Troisième étape}

Dans cette étape, nous allons donner une estimation a priori de $\|w\|_{L^{2}\left((0, t), L^{\infty}\right)}$ en appliquant les inégalités de Strichartz rappelées dans la proposition 1.3. La proposition 1.3 entraîne l'inégalité suivante, pour $0<t \leq T^{*}$,

$$
\begin{aligned}
\|w\|_{L^{2}\left((0, t), L^{\infty}\right)} \leq C_{4} & \left(\|w(0)\|_{1+\delta_{1}}+\left\|w_{t}(0)\right\|_{\delta_{1}}+\left\|\left(I-\tilde{S}_{0}\right) \mathbb{P}(w \nabla w)\right\|_{L^{1}\left((0, t), \dot{H}^{\delta_{1}}\right)}\right. \\
& +\left\|\left(I-\tilde{S}_{0}\right) \mathbb{P}(v \nabla v)\right\|_{L^{2}\left((0, t), \dot{H}^{1}\right)}+\left\|\left(I-\tilde{S}_{0}\right) \mathbb{P}(w \nabla v)\right\|_{L^{1}\left((0, t), \dot{H}^{\delta_{1}}\right)} \\
& \left.+\left\|\left(I-\tilde{S}_{0}\right) \mathbb{P}(v \nabla w)\right\|_{L^{1}\left((0, t), \dot{H}^{\delta_{1}}\right)}+\left\|\left(I-\tilde{S}_{0}\right) \mathbb{P} f\right\|_{L^{p}\left((0, t), \dot{H}^{\frac{p-1}{p}+d}\right)}\right),
\end{aligned}
$$

où $0<\delta_{1} \leq \delta, d>0$, où $1 \leq p \leq 2$ et où $\frac{3}{4}-\frac{1}{p}+\frac{p-1}{2 p}+\frac{d}{2}>0$. Il reste donc à majorer les normes des quatre termes quadratiques du membre droit de l'inégalité (2.35). Les inégalités (1.13), (1.16), la loi de produit (1.17) et l'estimation (2.23) impliquent, pour $0<t \leq T^{*}$,

$$
\begin{aligned}
\left\|\left(I-\tilde{S}_{0}\right) \mathbb{P}(w \nabla w)\right\|_{L^{1}\left((0, t), \dot{H}^{\delta_{1}}\right)} & \leq 2 C_{2} C_{3} C_{1}^{2+\left(\delta+\delta_{1}\right) / 2}\|w\|_{L^{2}\left((0, t), L^{\infty}\right)}\|w\|_{L^{2}\left((0, t), \dot{H}^{\delta+1}\right)} \\
& \leq 4 C_{2} C_{3} C_{1}^{2+\left(\delta+\delta_{1}\right) / 2} \nu^{-1 / 2} E_{0}^{1 / 2}\|w\|_{L^{2}\left((0, t), L^{\infty}\right)} .
\end{aligned}
$$

En appliquant la loi de produit (1.17), en tenant compte de la définition de $T^{*}$ et en utilisant l'inégalité (2.23), on estime le terme $\left\|\left(I-\tilde{S}_{0}\right) \mathbb{P}(v \nabla v)\right\|_{L^{2}\left((0, t), \dot{H}^{1}\right)}$ de la manière suivante, pour $0<t \leq T^{*}$,

$$
\begin{aligned}
\left\|\left(I-\tilde{S}_{0}\right) \mathbb{P}(v \nabla v)\right\|_{L^{2}\left((0, t), \dot{H}^{1}\right)} & \leq 2 C_{2}\left(\int_{0}^{t}\|v\|_{L^{\infty}}^{2}\|v\|_{2}^{2} d s\right)^{1 / 2} \leq 2 C_{2} C_{5}\left(\int_{0}^{t}\|v\|_{L^{\infty}}^{2}\|\Delta v\|_{2}^{2} d s\right)^{1 / 2} \\
& \leq 2 C_{2} C_{5}\|v\|_{L_{t}^{\infty}\left(L^{\infty}\right)}\|\Delta v\|_{L_{t}^{2}\left(L^{2}\right)} \leq 4 C_{2} C_{5} k_{0} \nu^{-1 / 2} E_{0}^{1 / 2}
\end{aligned}
$$

En utilisant la loi de produit (1.18) avec $u_{1}=w, u_{2}=\nabla v, s_{1}=\delta_{1}+1 / 2, s_{2}=1$ et les inégalités (1.13) et (2.23), on obtient, pour $0<t \leq T^{*}$,

$$
\begin{aligned}
\left\|\left(I-\tilde{S}_{0}\right) \mathbb{P}(w \nabla v)\right\|_{L^{1}\left((0, t), \dot{H}^{\delta_{1}}\right)} & \leq 4 C_{3}^{*} \int_{0}^{t}\|w\|_{\delta_{1}+1 / 2}\|\nabla v\|_{1} d s \leq 4 C_{3}^{*} C_{5} C_{1}^{\frac{7+2 \delta+2 \delta_{1}}{4}} \int_{0}^{t}\|w\|_{1+\delta}\|\Delta v\|_{L^{2}} d s \\
& \leq 4 C_{3}^{*} C_{5} C_{1}^{\frac{7+2 \delta+2 \delta_{1}}{4}}\|w\|_{L_{t}^{2}\left(\dot{H}^{\delta+1}\right)}\|\Delta v\|_{L_{t}^{2}\left(L^{2}\right)} \leq 16 C_{3}^{*} C_{5} C_{1}^{\frac{7+2 \delta+2 \delta_{1}}{4}} \nu^{-1} E_{0} .
\end{aligned}
$$

Il reste à majorer le terme $\left\|\left(I-\tilde{S}_{0}\right) \mathbb{P}(v \nabla w)\right\|_{L^{1}\left((0, t), \dot{H}^{\delta_{1}}\right)}$. Pour estimer ce terme, on va appliquer l'inégalité améliorée (1.19). En utilisant l'inégalité (1.19) avec $u_{1}=v, u_{2}=\nabla w, s_{1}=\frac{3}{2}+\delta_{1}-\delta, s_{2}=\delta$ et $p=6$, l'injection continue de $\dot{H}^{1}$ dans $L^{6}$, le lemme de Bernstein et les inégalités (1.13), on obtient grâce aux majorations (2.23) et $(2.33)$, pour $0<t \leq T^{*}$,

$$
\begin{aligned}
\left\|\left(I-\tilde{S}_{0}\right) \mathbb{P}(v \nabla w)\right\|_{L^{1}\left((0, t), \dot{H}^{\delta_{1}}\right)} & \leq \int_{0}^{t}\left(2 C_{3}^{*}\|v\|_{\delta_{1}-\delta+3 / 2}\|w\|_{1+\delta}+C_{6, j_{0}}\|v\|_{L^{6}}\|w\|_{1+\delta_{1}}\right) d s \\
& \leq \int_{0}^{t}\left(2 C_{3}^{*} C_{0} 2^{\left(\delta_{1}-\delta+1 / 2\right) j_{0}}+C_{6, j_{0}} C_{S} C_{1}^{2+\left(\delta_{1}+\delta\right) / 2}\right)\|\nabla v\|_{L^{2}}\|w\|_{1+\delta} d s \\
& \leq\left(2 C_{3}^{*} C_{0} 2^{\left(\delta_{1}-\delta+1 / 2\right) j_{0}}+C_{6, j_{0}} C_{S} C_{1}^{2+\left(\delta_{1}+\delta\right) / 2}\right)\|\nabla v\|_{L_{t}^{2}\left(L^{2}\right)}\|w\|_{L_{t}^{2}\left(\dot{H}^{\delta+1}\right)} \\
& \leq 4 \sqrt{2}\left(2 C_{3}^{*} C_{0} 2^{\left(\delta_{1}-\delta+1 / 2\right) j_{0}}+C_{6, j_{0}} C_{S} C_{1}^{2+\left(\delta_{1}+\delta\right) / 2}\right) \nu^{-1} E_{0}^{1 / 2}\left(E_{0}+E_{1}\right)^{1 / 2}
\end{aligned}
$$

Si on suppose que

$$
4 C_{2} C_{3} C_{4} C_{1}^{2+\left(\delta+\delta_{1}\right) / 2} \nu^{-1 / 2} E_{0}^{1 / 2} \leq \frac{1}{2}
$$


on peut déduire des majorations (2.35), (2.36), (2.37), (2.38) et (2.39) que, pour $0<t \leq T^{*}$,

$$
\begin{aligned}
& \|w\|_{L^{2}\left((0, t), L^{\infty}\right)} \leq 2 C_{4}\left(4 C_{2} C_{5} k_{0} \nu^{-1 / 2} E_{0}^{1 / 2}+16 C_{3}^{*} C_{5} C_{1}^{\frac{7+2 \delta+2 \delta_{1}}{4}} \nu^{-1} E_{0}\right. \\
& +4 \sqrt{2}\left(2 C_{3}^{*} C_{0} 2^{\left(\delta_{1}-\delta+1 / 2\right) j_{0}}+C_{6, j_{0}} C_{S} C_{1}^{2+\left(\delta_{1}+\delta\right) / 2}\right) \nu^{-1} E_{0}^{1 / 2}\left(E_{0}+E_{1}\right)^{1 / 2}
\end{aligned}
$$

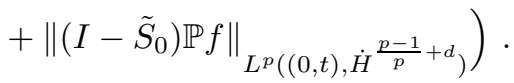

\subsection{Quatrième étape}

Dans cette étape, on montre tout d'abord que $T^{*} \geq T_{0}$. On rappelle que $k_{0}>0$ est un nombre réel fixé satisfaisant aux conditions (2.21). Les inégalités (2.23), (2.33), (2.34) et (2.41) impliquent, pour $0<t \leq T^{*}$,

$$
\|v(t)\|_{L^{\infty}}+\|\Delta v\|_{L^{2}\left((0, t), L^{2}\right)}^{1 / 2}\|\nabla v\|_{L^{2}\left((0, t), L^{2}\right)}^{1 / 2}+\|w\|_{L^{2}\left((0, t), L^{\infty}\right)} \leq B
$$

où

$$
\begin{aligned}
B= & \left.4 C_{A}\left(\frac{8}{\nu}\right)^{3 / 8} E_{0}^{3 / 8}\left(E_{0}+E_{1}\right)^{1 / 8}+4 \sqrt{2} \nu^{-1} E_{0}^{1 / 2}\left(E_{0}+E_{1}\right)^{1 / 2}+2 C_{4}\left\|\left(I-\tilde{S}_{0}\right) \mathbb{P} f\right\|_{L^{p}((0, t), \dot{H}} \frac{p-1}{p}+d\right) \\
+ & 2 C_{4}\left[4 C_{2} C_{5} k_{0} \nu^{-1 / 2} E_{0}^{1 / 2}+16 C_{3}^{*} C_{5} C_{1}^{\frac{7+2 \delta+2 \delta_{1}}{4}} \nu^{-1} E_{0}\right. \\
& \left.+4 \sqrt{2}\left(2 C_{3}^{*} C_{0} 2^{\left(\delta_{1}-\delta+1 / 2\right) j_{0}}+C_{6, j_{0}} C_{S} C_{1}^{2+\left(\delta_{1}+\delta\right) / 2}\right) \nu^{-1} E_{0}^{1 / 2}\left(E_{0}+E_{1}\right)^{1 / 2}\right] .
\end{aligned}
$$

Si on choisit maintenant les conditions initiales $\left(u_{0}, u_{1}\right)$ et la force $f$ suffisamment petites, c'est-à-dire $f, E_{0}$ et le produit $E_{0} E_{1}$ suffisamment petits pour que

$$
\begin{aligned}
& 4 C_{A}\left(\frac{8}{\nu}\right)^{3 / 8} E_{0}^{3 / 8}\left(E_{0}+E_{1}\right)^{1 / 8}+4 \sqrt{2} \nu^{-1} E_{0}^{1 / 2}\left(E_{0}+E_{1}\right)^{1 / 2}+2 C_{4}\left[4 C_{2} C_{5} k_{0} \nu^{-1 / 2} E_{0}^{1 / 2}+16 C_{3}^{*} C_{5} C_{1}^{\frac{7+2 \delta+2 \delta_{1}}{4}} \nu^{-1} E_{0}\right. \\
& \left.\left.+4 \sqrt{2}\left(2 C_{3}^{*} C_{0} 2^{\left(\delta_{1}-\delta+1 / 2\right) j_{0}}+C_{6, j_{0}} C_{S} C_{1}^{2+\left(\delta_{1}+\delta\right) / 2}\right) \nu^{-1} E_{0}^{1 / 2}\left(E_{0}+E_{1}\right)^{1 / 2}+\left\|\left(I-\tilde{S}_{0}\right) \mathbb{P} f\right\|_{L^{p}(\dot{H}} \frac{p-1}{p}+d\right)\right] \leq \frac{k_{0}}{2},
\end{aligned}
$$

alors, la majoration (2.43) entraîne que, pour $0 \leq t \leq T^{*}$,

$$
\|v(t)\|_{L^{\infty}}+\|\Delta v\|_{L^{2}\left((0, t), L^{2}\right)}^{1 / 2}\|\nabla v\|_{L^{2}\left((0, t), L^{2}\right)}^{1 / 2}+\|w\|_{L^{2}\left((0, t), L^{\infty}\right)} \leq \frac{k_{0}}{2}
$$

ce qui contredit la définition de $T^{*}$. Donc on a montré par contradiction que $T^{*} \geq T_{0}$. Mais alors les majorations (2.23) et $(2.33)$ montrent que $\left\|\left(u, u_{t}\right)(t)\right\|_{\dot{H}^{\delta+1}}$ reste borné sur $\left[0, T_{0}\right]$, ce qui contredit le fait que $T_{0}$ soit fini. Donc $T_{0}$ est infini et on a démontré l'existence globale de solutions.

Si on traduit la condition (2.44) dans les variables temporelle $\tau$ et spatiales $y$ de départ et si on tient compte des propriétés (1.4), (1.5) et (1.6), on obtient directement le théorème 0.4.

\section{A. Annexe A}

Dans cette annexe, nous démontrerons les inégalités (1.17) et (1.19) de la proposition 1.2. Pour démontrer ces inégalités, on utilise les lois du paraproduit introduit par Bony [2]. Dans les démonstrations suivantes, $C$ et $\tilde{C}$ désignent des constantes strictement positives génériques. On rappelle d'abord qu'il existe une constante 
strictement positive $C_{6}$ telle que, pour tout $s \in \mathbb{R}$, pour tout $u \in \dot{H}^{s}(\mathbb{R})$,

$$
C_{6}^{-s-1}\|u\|_{\dot{H}^{s}}^{2} \leq \sum_{j \in \mathbb{Z}} 2^{2 j s}\left\|\dot{\Delta}_{j} u\right\|_{L^{2}}^{2} \leq C_{6}^{s+1}\|u\|_{\dot{H}^{s}}^{2}
$$

où

$$
\begin{aligned}
\dot{\Delta}_{j} u & =\mathcal{F}^{-1}\left(\varphi_{j}(\xi) \mathcal{F}(u)(\xi)\right), \quad \forall j \in \mathbb{Z} \\
\dot{S}_{q} u & =\sum_{j \leq q-1} \dot{\Delta}_{j} u, \quad \forall q, q \in \mathbb{Z} .
\end{aligned}
$$

On vérifie facilement que, avec le choix des fonctions $\varphi$ et $\chi$, fait au paragraphe 1.2, on a l'égalité suivante (voir [2], [6] et [17]),

$$
\begin{aligned}
\dot{\Delta}_{j}\left(u_{1} \nabla u_{2}\right)= & \dot{\Delta}_{j}\left(\sum_{\left|j-j^{\prime}\right| \leq 4} \dot{S}_{j^{\prime}-1} u_{1} \dot{\Delta}_{j^{\prime}}\left(\nabla u_{2}\right)+\sum_{\left|j-j^{\prime}\right| \leq 4} \dot{S}_{j^{\prime}-1}\left(\nabla u_{2}\right) \dot{\Delta}_{j^{\prime}} u_{1}\right. \\
& \left.+\sum_{\left|j^{\prime}\right| \geq j-4} \sum_{i \in\{0, \pm 1\}} \dot{\Delta}_{j^{\prime}} u_{1} \dot{\Delta}_{j^{\prime}+i}\left(\nabla u_{2}\right)\right) .
\end{aligned}
$$

On suppose dorénavant que $s \geq 0$. L'inégalité (1.12) du lemme de Bernstein nous permet de majorer le terme $\left\|\dot{\Delta}_{j}\left(\sum_{\left|j-j^{\prime}\right| \leq 4} \dot{S}_{j^{\prime}-1} u_{1} \dot{\Delta}_{j^{\prime}}\left(\nabla u_{2}\right)\right)\right\|_{L^{2}}$ de la manière suivante:

$$
\begin{aligned}
\left\|\dot{\Delta}_{j}\left(\sum_{\left|j-j^{\prime}\right| \leq 4} \dot{S}_{j^{\prime}-1} u_{1} \dot{\Delta}_{j^{\prime}}\left(\nabla u_{2}\right)\right)\right\|_{L^{2}} & \leq C \sum_{\left|j-j^{\prime}\right| \leq 4}\left\|u_{1}\right\|_{L^{\infty}}\left\|\dot{\Delta}_{j^{\prime}}\left(\nabla u_{2}\right)\right\|_{L^{2}} \\
& \leq C C_{0}\left\|u_{1}\right\|_{L^{\infty}} \sum_{\left|j-j^{\prime}\right| \leq 4} 2^{j^{\prime}}\left\|\dot{\Delta}_{j^{\prime}} u_{2}\right\|_{L^{2}} \\
& \leq \tilde{C}\left\|u_{1}\right\|_{L^{\infty}}\left\|u_{2}\right\|_{s+1} 2^{-s j} a_{j}
\end{aligned}
$$

où $a_{j}$ est une série de nombres positifs de carré sommable (c'est-à-dire $\left.\sum_{j \in \mathbb{Z}} a_{j}^{2}<+\infty\right)$. Les inégalités (1.10) et (1.12) du lemme de Bernstein entraînent aussi que

$$
\begin{aligned}
\left\|\dot{\Delta}_{j}\left(\sum_{\left|j-j^{\prime}\right| \leq 4} \dot{S}_{j^{\prime}-1}\left(\nabla u_{2}\right) \dot{\Delta}_{j^{\prime}} u_{1}\right)\right\|_{L^{2}} & \leq C \sum_{\left|j-j^{\prime}\right| \leq 4}\left\|\dot{S}_{j^{\prime}-1}\left(\nabla u_{2}\right)\right\|_{L^{\infty}}\left\|\dot{\Delta}_{j^{\prime}} u_{1}\right\|_{L^{2}} \\
& \leq C C_{0} \sum_{\left|j-j^{\prime}\right| \leq 4} 2^{j^{\prime}-2}\left\|\dot{S}_{j^{\prime}-1} u_{2}\right\|_{L^{\infty}}\left\|\dot{\Delta}_{j^{\prime}} u_{1}\right\|_{L^{2}} \\
& \leq C\left\|u_{2}\right\|_{L^{\infty}} \sum_{\left|j-j^{\prime}\right| \leq 4}\left\|\dot{\Delta}_{j^{\prime}}\left(\nabla u_{1}\right)\right\|_{L^{2}} \\
& \leq \tilde{C}\left\|u_{2}\right\|_{L^{\infty}}\left\|u_{1}\right\|_{s+1} 2^{-s j} b_{j}
\end{aligned}
$$

où $b_{j}$ est une série de nombres positifs de carré sommable. 
Il reste à majorer le terme $\left\|\dot{\Delta}_{j}\left(\sum_{j^{\prime} \geq j-4} \sum_{i \in\{0, \pm 1\}} \dot{\Delta}_{j^{\prime}} u_{1} \dot{\Delta}_{j^{\prime}+i}\left(\nabla u_{2}\right)\right)\right\|_{L^{2}}$. Grâce au lemme de Bernstein, on peut écrire:

$$
\begin{aligned}
\left\|\dot{\Delta}_{j}\left(\sum_{j^{\prime} \geq j-4} \sum_{i \in\{0, \pm 1\}} \dot{\Delta}_{j^{\prime}} u_{1} \dot{\Delta}_{j^{\prime}+i}\left(\nabla u_{2}\right)\right)\right\|_{L^{2}} & =\left\|\sum_{j^{\prime} \geq j-4} \sum_{i \in\{0, \pm 1\}} \dot{\Delta}_{j}\left(\dot{\Delta}_{j^{\prime}} u_{1} \dot{\Delta}_{j^{\prime}+i}\left(\nabla u_{2}\right)\right)\right\|_{L^{2}} \\
& \leq C C_{0}\left\|u_{1}\right\|_{L^{\infty}} \sum_{j^{\prime} \geq j-4} \sum_{i \in\{0, \pm 1\}} 2^{j^{\prime}+i}\left\|\dot{\Delta}_{j^{\prime}+i} u_{2}\right\|_{L^{2}} \\
& \leq \tilde{C} 2^{-s j}\left\|u_{1}\right\|_{L^{\infty}}\left\|u_{2}\right\|_{s+1} 2^{s j}\left(\sum_{j^{\prime} \geq j-4} 2^{-s j^{\prime}} c_{j^{\prime}}\right)
\end{aligned}
$$

où $c_{j^{\prime}}$ est une série de nombres positifs de carré sommable. On pose

$$
\left.d_{j}=\sum_{j^{\prime} \geq j-4} 2^{-s\left(j^{\prime}-j\right)} c_{j^{\prime}}=\left(\chi_{\left\{j^{\prime} \leq 4\right\}} 2^{s j^{\prime}}\right) * c_{j^{\prime}}\right)(j) .
$$

L'inégalité de Young implique que $d_{j}$ est de carré sommable. En effet, on a,

$$
\left(\sum_{j \in \mathbb{Z}} d_{j}^{2}\right)^{1 / 2} \leq\left\|\chi_{\left\{j^{\prime} \leq 4\right\}} 2^{s j^{\prime}}\right\|_{\ell^{1}(\mathbb{N})}\left\|c_{j}\right\|_{\ell^{2}(\mathbb{Z})} \leq \frac{\tilde{C}}{s} .
$$

Si on tient compte des inégalités (A.1), qui nous donnent une expression équivalente de $\left\|u_{1} \nabla u_{2}\right\|_{s}$, et si on applique l'égalité (A.3), on déduit immédiatement des inégalités (A.4) à (A.7) que

$$
\left\|u_{1} \nabla u_{2}\right\|_{s} \leq C_{3}\left(\left\|u_{1}\right\|_{L^{\infty}}\left\|u_{2}\right\|_{s+1}+\left\|u_{2}\right\|_{L^{\infty}}\left\|u_{1}\right\|_{s+1}\right)
$$

qui est précisément l'inégalité (1.17).

L'inégalité (1.18) est classique (pour une démonstration, voir, par exemple, [6]). On peut aussi trouver une démonstration dans [17]. Supposons maintenant que $s_{1}+s_{2}-3 / 2 \geq 0$. Les démonstrations dans ces deux références impliquent aussi, après utilisation des propriétés (A.1) et (A.3), que

$$
\begin{aligned}
\left\|u_{1} u_{2}\right\|_{s_{1}+s_{2}-3 / 2}^{2} & \leq \sum_{j \in \mathbb{Z}} 2^{2 j\left(s_{1}+s_{2}-3 / 2\right)}\left\|\dot{\Delta}_{j}\left(u_{1} u_{2}\right)\right\|_{L^{2}}^{2} \\
& \leq C \sum_{j \in \mathbb{Z}} 2^{2 j\left(s_{1}+s_{2}-3 / 2\right)}\left(\left\|\dot{\Delta}_{j}\left(\sum_{\left|j-j^{\prime}\right| \leq 4} \dot{S}_{j^{\prime}-1} u_{2} \dot{\Delta}_{j^{\prime}} u_{1}\right)\right\|_{L^{2}}^{2}\right. \\
& \left.+\left\|\dot{\Delta}_{j}\left(\sum_{\left|j^{\prime}\right| \geq j-4} \sum_{i \in\{0, \pm 1\}} \dot{\Delta}_{j^{\prime}} u_{1} \dot{\Delta}_{j^{\prime}+i} u_{2}\right)\right\|_{L^{2}}^{2}\right) \\
& +C \sum_{j \in \mathbb{Z}} 2^{2 j\left(s_{1}+s_{2}-3 / 2\right)}\left\|\dot{\Delta}_{j}\left(\sum_{\left|j-j^{\prime}\right| \leq 4} \dot{S}_{j^{\prime}-1} u_{1} \dot{\Delta}_{j^{\prime}} u_{2}\right)\right\|_{L^{2}}^{2} \\
& \leq C\left(\frac{1}{s_{1}+s_{2}}+\frac{1}{3 / 2-s_{2}}\right)\left\|u_{1}\right\|_{s_{1}}^{2}\left\|u_{2}\right\|_{s_{2}}^{2} \\
& +C \sum_{j \in \mathbb{Z}} 2^{2 j\left(s_{1}+s_{2}-3 / 2\right)}\left\|\dot{\Delta}_{j}\left(\sum_{\left|j-j^{\prime}\right| \leq 4} \dot{S}_{j^{\prime}-1} u_{1} \dot{\Delta}_{j^{\prime}} u_{2}\right)\right\|_{L^{2}}^{2}
\end{aligned}
$$


Puisque le support de la transformée de Fourier de $u_{1}$ est inclus dans la réunion finie de couronnes $\cup_{-1 \leq j \leq q} 2^{j} \mathcal{C}$, où $q \in \mathbb{N}$, on peut écrire, grâce au lemme de Bernstein que

$$
\begin{aligned}
\sum_{j \in \mathbb{Z}} 2^{2 j\left(s_{1}+s_{2}-3 / 2\right)}\left\|\dot{\Delta}_{j}\left(\sum_{\left|j-j^{\prime}\right| \leq 4} \dot{S}_{j^{\prime}-1} u_{1} \dot{\Delta}_{j^{\prime}} u_{2}\right)\right\|_{L^{2}}^{2} & \leq C \sum_{j \in \mathbb{Z}} 2^{2 j\left(s_{1}+s_{2}-3 / 2\right)}\left(\sum_{\left|j-j^{\prime}\right| \leq 4}\left\|\dot{S}_{j^{\prime}-1} u_{1}\right\|_{L^{\infty}}^{2}\left\|\dot{\Delta}_{j^{\prime}} u_{2}\right\|_{L^{2}}^{2}\right) \\
& \leq C C_{0} \sum_{j \in \mathbb{Z}} 2^{2 j\left(s_{1}+s_{2}-3 / 2\right)} \times 2^{6 q / p}\left\|u_{1}\right\|_{L^{p}}^{2} \sum_{\left|j-j^{\prime}\right| \leq 4}\left\|\dot{\Delta}_{j^{\prime}} u_{2}\right\|_{L^{2}}^{2} \\
& \leq \tilde{C} 2^{6 q / p}\left\|u_{1}\right\|_{L^{p}}^{2}\left\|u_{2}\right\|_{s_{1}+s_{2}-3 / 2}^{2} .
\end{aligned}
$$

L'inégalité (1.19) est une conséquence directe de (A.8) et de (A.9).

\section{REFERENCES}

[1] S. Alinhac et P. Gérard, Opérateurs pseudo-différentiels et théorème de Nash-Moser Savoirs Actuels. InterEditions, Paris; Éditions du Centre National de la Recherche Scientifique (CNRS), Meudon, (1991).

[2] J.-M. Bony, Calcul symbolique et propagation des singularités pour les équations aux dérivées partielles non linéaires, Ann. Sci. Ec. Norm. Sup., 14 (1981), pages 209-246.

[3] Y. Brenier, R. Natalini et M. Puel, On a relaxation approximation of the incompressible Navier-Stokes equations, Proc. Amer. Math. Soc. 132 (2004), pages 1021-1028.

[4] C. Cattaneo, Sulla conduzione del calore, Atti Sem. Mat. Fis. Univ. Modena 3, (1949), pages 83-101.

[5] _ Sur une forme de l'équation de la chaleur éliminant le paradoxe d'une propagation instantanée, C. R. Acad. Sci. Paris 247, (1958), pages 431-433.

[6] J.-Y. Chemin, Fluides parfaits incompressibles. Astérisque No. 230 (1995).

[7] J. K. Hale et G. Raugel, Upper semicontinuity of the attractor for a singularly perturbed hyperbolic equation, J. Differential Equations 73 (1988), pages 197-214.

[8] L Lower Semicontinuity of the attractor for a singularly perturbed hyperbolic equation, J. Dyn. Diff. Equations 2 (1990), pages $19-67$.

[9] E.C. Hunke et J.R. Dukowicz, 1997: An elastic-viscous-plastic model for sea ice dynamics, J. Phys. Oceanogr., 27 (1997), pages 1849-1868.

[10] T.Katsaounis et C. Makridakis, Relaxation models and finite element schemes for the shallow water equations, Hyperbolic problems: theory, numerics, applications, Springer, Berlin, (2003), pages 621-631.

[11] T. Katsaounis, C. Makridakis et C. Simeoni Stability and convergence of relaxation finite element schemes for the incompressible Navier-Stokes equations, Proceedings of the Conference on Hyperbolic Problems, Yakohama Pupl., (2005).

[12] J.-L. Lions, Quelques méthodes de résolution des problèmes aux limites non linéaires, Gauthier Villars, Paris, 1969.

[13] B. Martuzans, A. Terentyev et Yu. Skryl, Derivation of hyperbolic transfer equations from first principles, Latvian Journal of Physics and Technical Sciences 1 (2005), pages 23-33.

[14] K. Mischaikow et G. Raugel Singularly perturbed partial differential equations and stability of the Conley index, manuscrit.

[15] R. Natalini et F. Rousset Convergence of a singular Euler-Poisson approximation of the incompressible Navier-Stokes equations, Proc. Am. Math. Soc. 134 (2006), pages 2251-2258.

[16] M. Paicu et G. Raugel A hyperbolic singular perturbation of the Navier-Stokes equations in $\mathbf{R}^{2}$, manuscrit.

[17] M. Paicu et G. Raugel Equations de Navier-Stokes, cours de M2, Université Paris-Sud, manuscrit.

[18] G. Raugel Singularly perturbed hyperbolic equations revisited, Fiedler, B. (ed.) et al., International conference on differential equations. Proceedings of the conference, Equadiff '99, Berlin, Germany, 1999. Vol. 1. Singapore: World Scientific, (2000), pages 647-652 .

[19] A. Terentyev et Yu. Skryl, Derivation of hyperbolic transfer equations from BGK-Equation, arXiv:cond-mat/0507333 (2005). 